\title{
Theoretical Studies on the Effects of Dispersal Corridors on the Permanence of Discrete Predator-Prey Models in Patchy Environment
}

\author{
Chunqing Wu, ${ }^{1}$ Shengming Fan, ${ }^{1}$ and Patricia J. Y. Wong ${ }^{2}$ \\ ${ }^{1}$ School of Mathematics and Physics, Changzhou University, Changzhou 213164, China \\ ${ }^{2}$ School of Electrical and Electronic Engineering, Nanyang Technological University, Singapore 639798 \\ Correspondence should be addressed to Patricia J. Y. Wong; ejywong@ntu.edu.sg
}

Received 25 March 2014; Revised 11 June 2014; Accepted 12 June 2014; Published 17 July 2014

Academic Editor: Josef Diblík

Copyright (C) 2014 Chunqing Wu et al. This is an open access article distributed under the Creative Commons Attribution License, which permits unrestricted use, distribution, and reproduction in any medium, provided the original work is properly cited.

We study two discrete predator-prey models in patchy environment, one without dispersal corridors and one with dispersal corridors. Dispersal corridors are passes that allow the migration of species from one patch to another and their existence may influence the permanence of the model. We will offer sufficient conditions to guarantee the permanence of the two predator-prey models. By comparing the two permanence criteria, we discuss the effects of dispersal corridors on the permanence of the predatorprey model. It is found that the dispersion of the prey from one patch to another is helpful to the permanence of the prey if the population growth of the prey is density dependent; however, this dispersion of the prey could be disadvantageous or advantageous to the permanence of the predator. Five numerical examples are presented to confirm the theoretical results obtained and to illustrate the effects of dispersal corridors on the permanence of the predator-prey model.

\section{Introduction}

The conception of permanence in ecological communities is first discussed in [1]. This notion of permanence is also known under different aliases, such as permanent coexistence or uniform persistence. Aptly termed, permanence of a population model reflects that all the species in the ecosystem will coexist for a relatively long time; that is, no extinction of any species will occur in the near future-this is very important in biological conservation. A permanent model may attain globally asymptotically stable positive equilibrium [2] or may have periodic solution [3, 4], and even a chaotic model can be permanent [5]. The sufficient and/or necessary conditions established to guarantee the permanence of various population models may offer helpful suggestions for environmental managers to take applicable measures for the sustainability of the ecosystems [6-8].

It is common that in the real world today the habitats of the species are separated into isolated patches due to spatial heterogeneity or the development of mankind, such as the construction of highways or railways [9-16]. This patchy environment may restrict the activities of the species and lead to adverse effects on the permanence of the ecological communities; therefore, some dispersal corridors may be built to allow movement between the patches. For example, the construction of Qinghai-Tibet railway in China separates the habitats of the Tibetan antelopes to pieces of small patches. In order to reduce the impact of patchy environment on the Tibetan antelopes, several underpasses (dispersal corridors) along the railway are constructed for the Tibetan antelopes to move from one side of the railway to the other side [17]. Indeed some researches [18] have verified that the Tibetan antelopes have really made use of these underpasses to cross the railway.

In the literature there are quite many papers that discuss the permanence of continuous time predator-prey or competitive systems in patchy environment: for example, one can refer to [19-23] and the many references cited therein. Though some continuous time models have their discrete time analogues, it is common knowledge that these discrete 
time analogues may present different dynamics, which are possibly more complicated [24-26]. To our best knowledge, there are few references on discrete time predator-prey or competitive models in patchy environment; see, for instance, $[22,27-30]$. In $[28,30]$, the effects of dispersion on discrete models for single species in patchy environment have been discussed; two patches have been considered in [30] while $n$ patches have been tackled in [28]. In [27, 29], the effects of dispersion on discrete competitive models in patchy environment have been analyzed; in particular discrete Leslie-Gower type competitive model has been adopted in [27]. Motivated by the above papers as well as the importance of permanence in ecosystems, in this paper we will investigate the permanence of discrete time predator-prey models of Ricker type in patchy environment. To be specific, we will tackle two discrete predator-prey models in patchy environment; the first model has no dispersal corridors while the second model has dispersal corridors. Sufficient conditions to guarantee permanence will be established for both models. The effects of dispersal corridors on permanence are then analyzed by comparing the two sets of sufficient conditions. We remark that in the literature the numerous research papers on continuous/discrete predator-prey models in patchy environment have focused mainly on the permanence criteria and/or the existence of periodic solutions of the models; however, the effects of dispersal corridors on the permanence of the models are rarely studied until the present paper.

The paper is organized as follows. In Section 2, we construct the two discrete predator-prey models in patchy environment with/without dispersal corridors; also in this section we give some preliminaries such as the definition of permanence. In Section 3, sufficient conditions for the permanence of the two discrete predator-prey models in patchy environment with/without dispersal corridors are offered. The effects of dispersal corridors on the permanence of the models are analyzed in Section 4. In Section 5, we present five examples where numerical simulations are performed to confirm the theoretical results and to illustrate the effects of dispersal corridors. Finally, we conclude in Section 6. The mathematical proofs of the theorems in Section 3 are presented in Appendix.

\section{Models and Preliminaries}

There are many types of discrete time mathematical models, which are governed by difference equations, established to reflect the fluctuation of the single-species population [31]. Among them, the Ricker type model, given as

$$
x(n+1)=x(n) \exp [r-a x(n)],
$$

is first established for some kind of fishery population [32] and subsequently used to model other kinds of single-species population [31, 33, 34]. In model $(1), x(n)$ is the population density of the species $x$ at the $n$th time step, $r$ is the intrinsic growth rate of $x, a$ is the strength of intraspecific competition of $x$, and $r, a$ are positive constants. Model (1) is logistic; that is, the fluctuation of the population of $x$ is undergoing the effect of density dependency. One can refer to [31] for more details about (1).
In the predator-prey model that we are considering in this paper, the prey is $x$ and the predator is $y$, and the predator $y$ only feeds on the prey $x$. Adopting model (1) to reflect the population growth of the prey $x$, we have the following discrete predator-prey model of Lotka-Volterra type:

$$
\begin{aligned}
& x(n+1)=x(n) \exp \left[r_{1}-a_{11} x(n)-a_{12} y(n)\right], \\
& y(n+1)=y(n) \exp \left[-r_{2}+a_{21} x(n)-a_{22} y(n)\right] .
\end{aligned}
$$

In model (2), $r_{1}$ is the intrinsic growth rate of the prey $x, a_{11}$ is the intraspecific competition strength of the prey $x, a_{12}$ is the predation strength, $r_{2}$ is the death rate of the predator $y$ if there is no prey $x$ for $y$ to feed on, $a_{21}$ reflects the predation efficiency or the rate of energy transferred from the prey $x$ to the predator $y$ through predation, and $a_{22}$ is the intraspecific competition strength of the predator $y$. The parameters $r_{i}, a_{i j}$, $i$, and $j \in\{1,2\}$ are all positive constants. The permanence and the global asymptotic stability of the positive equilibrium of model (2) have been discussed in $[35,36]$.

Now, suppose that the habitats of the predator-prey system (2) are separated into two patches, patch 1 and patch 2. We denote $x_{i}(n)$ and $y_{i}(n)$ as the respective population density of the prey $x$ and the predator $y$ in patch $i$ at the $n$th time step, $i \in\{1,2\}$. Let $r_{1 i}$ be the intrinsic growth rate of the prey $x$ in patch $i$ and let $r_{2 i}$ be the death rate of the predator $y$ in patch $i, i \in\{1,2\}$. Also, $a_{i j}^{(k)}$ has the same biological meaning as $a_{i j}$ in model (2) except that now it is the case in patch $k, i, j, k \in\{1,2\}$. At this moment, there are no dispersal corridors between the two patches. Thus, we have the following discrete predator-prey model in patchy environment without dispersal corridors:

$$
\begin{aligned}
& x_{1}(n+1)=x_{1}(n) \exp \left[r_{11}-a_{11}^{(1)} x_{1}(n)-a_{12}^{(1)} y_{1}(n)\right], \\
& y_{1}(n+1)=y_{1}(n) \exp \left[-r_{21}+a_{21}^{(1)} x_{1}(n)-a_{22}^{(1)} y_{1}(n)\right], \\
& x_{2}(n+1)=x_{2}(n) \exp \left[r_{12}-a_{11}^{(2)} x_{2}(n)-a_{12}^{(2)} y_{2}(n)\right], \\
& y_{2}(n+1)=y_{2}(n) \exp \left[-r_{22}+a_{21}^{(2)} x_{2}(n)-a_{22}^{(2)} y_{2}(n)\right] .
\end{aligned}
$$

Next, we introduce dispersal corridors to the patchy model (3). It is assumed that the prey $x$ can migrate from one patch to another via these dispersal corridors, but the predator $y$ cannot use the dispersal corridors to migrate. We make some remarks to this assumption. Firstly, this assumption of only prey (but not predator) dispersal is usual in continuous/discrete time predator-prey models in patchy environment; see, for example, [22, 23, 37, 38]. Moreover, this assumption fits the common definition of prey refuge if the predator is fixed in one patch, and intensive studies have been done; refer to [39] and the references cited therein. Our assumption here is that the prey can move among patches while the predator is fixed in each patch-an example of such a system can be found in [40], where the prey (mayflies) is mobile among different patches, but the predator (charr) is confined to its patch since the river is fenced into patches. Indeed, systems of this type may exist naturally in swamp fields, where the prey (such as flying insects) can migrate 
to different ponds while the predator (such as fish) is fixed in each pond. Denote $d_{i j}$ as the dispersal rate of the prey $x$ migrating from patch $i$ to patch $j, i, j \in\{1,2\}, i \neq j$. We have the following discrete predator-prey model in patchy environment with dispersal corridors:

$$
\begin{array}{r}
x_{1}(n+1) \\
=x_{1}(n) \exp \left[r_{11}-a_{11}^{(1)} x_{1}(n)-a_{12}^{(1)} y_{1}(n)\right. \\
\left.-d_{12} x_{1}(n)+d_{21} x_{2}(n)\right],
\end{array}
$$$$
y_{1}(n+1)
$$$$
=y_{1}(n) \exp \left[-r_{21}+a_{21}^{(1)} x_{1}(n)-a_{22}^{(1)} y_{1}(n)+\alpha d_{21} x_{2}(n)\right],
$$$$
x_{2}(n+1)
$$$$
=x_{2}(n) \exp \left[r_{12}-a_{11}^{(2)} x_{2}(n)-a_{12}^{(2)} y_{2}(n)\right.
$$$$
\left.+d_{12} x_{1}(n)-d_{21} x_{2}(n)\right]
$$

$$
\begin{aligned}
& y_{2}(n+1) \\
& \quad=y_{2}(n) \exp \left[-r_{22}+a_{21}^{(2)} x_{2}(n)-a_{22}^{(2)} y_{2}(n)+\beta d_{12} x_{1}(n)\right] .
\end{aligned}
$$

Here, $\alpha$ and $\beta$ represent the predation rates of the predator on the prey that migrates from another patch. Note that model (4) is reduced to model (3) if the dispersal rates $d_{12}$ and $d_{21}$ are both zero. Model (4) may be viewed as a discrete analogue of the continuous time predator-prey reaction-diffusion model for patchy environment proposed by Allen [41]. In [41] an extinction result, whereby some sufficient conditions are established to ensure the extinction of the species, has been developed. We remark that, from a practical standpoint, permanence results that guarantee species survival are more useful than extinction results; as such, we will focus on the permanence of the discrete model.

In view of the biological background, we let the initial values of models (3) and (4) be

$$
\begin{array}{ll}
x_{1}(0)=x_{1}>0, & x_{2}(0)=x_{2}>0 ; \\
y_{1}(0)=y_{1}>0, & y_{2}(0)=y_{2}>0 .
\end{array}
$$

Also, throughout the paper we assume the following:

$$
\begin{gathered}
\left(H_{1}\right) r_{i j}, a_{i j}, a_{i j}^{(k)}, i, j, k \in\{1,2\} \text { and } \alpha, \beta \text { are all positive } \\
\text { constants, and } d_{12}, d_{21} \text { are nonnegative constants. }
\end{gathered}
$$

It is obvious that all the solutions of either model (3) or model (4) with initial values (5) are positive, which corresponds well to the biological meanings of $x_{i}(n), y_{i}(n), i \in$ $\{1,2\}$, noting that population density of any species is always nonnegative.

The permanence results of models (3) and (4) will be presented in Section 3. To be precise, we state the definition of permanence as follows.
Definition 1. Model (3) (or (4)) with initial values (5) is said to be permanent if there exist positive constants $m_{i}, m_{i}^{*}, M_{i}$, and $M_{i}^{*}, i \in\{1,2\}$, such that

$$
\begin{aligned}
& m_{i} \leq \liminf _{n \rightarrow+\infty} x_{i}(n) \leq \limsup _{n \rightarrow+\infty} x_{i}(n) \leq M_{i}, \quad i=1,2, \\
& m_{i}^{*} \leq \liminf _{n \rightarrow+\infty} y_{i}(n) \leq \limsup _{n \rightarrow+\infty} y_{i}(n) \leq M_{i}^{*}, \quad i=1,2,
\end{aligned}
$$

hold for each solution of model (3) (or (4)) with initial values (5).

\section{Permanence of the Models}

In this section, we will establish the permanence of models (3) and (4) with initial values (5). The mathematical proofs of Theorems 2, 4, and 5 will be presented in Appendix.

3.1. Permanence without Dispersal Corridors. We consider model (3) together with initial values (5). Notice that in (3) the first two equations are independent of the last two equations; therefore, we only need to consider the permanence of the model comprising just the first two equations of (3); namely,

$$
\begin{aligned}
& x_{1}(n+1)=x_{1}(n) \exp \left[r_{11}-a_{11}^{(1)} x_{1}(n)-a_{12}^{(1)} y_{1}(n)\right], \\
& y_{1}(n+1)=y_{1}(n) \exp \left[-r_{21}+a_{21}^{(1)} x_{1}(n)-a_{22}^{(1)} y_{1}(n)\right]
\end{aligned}
$$

with initial values

$$
x_{1}(0)=x_{1}>0, \quad y_{1}(0)=y_{1}>0 .
$$

Theorem 2. Suppose that

$$
\begin{aligned}
& r_{11}-a_{12}^{(1)} M_{1}>0, \\
& -r_{21}+a_{21}^{(1)} m_{1}>0
\end{aligned}
$$

are satisfied, where

$$
\begin{gathered}
M_{1}=\frac{1}{a_{22}^{(1)}} \exp \left(-r_{21}+a_{21}^{(1)} A_{1}-1\right), \\
A_{1}=\frac{1}{a_{11}^{(1)}} \exp \left(r_{11}-1\right), \\
m_{1}=\frac{r_{11}-a_{12}^{(1)} M_{1}}{a_{11}^{(1)}} \exp \left(r_{11}-a_{12}^{(1)} M_{1}-a_{11}^{(1)} A_{1}\right) .
\end{gathered}
$$

Then, model (7) with initial values (8) is permanent.

Remark 3. (a) The permanence or global asymptotical stability for the positive equilibrium of model (7) has been studied in $[35,36,42]$. In [35] it is shown that, for sufficiently small $r_{11}$ and $r_{21}$, model (7) has a positive equilibrium which is globally attractive, and as a consequence model (7) is permanent. So the conditions for the permanence of (7) are not directly derived in [35]. On the other hand, based on the study of a nonautonomous version of (7), it is proved in [36] (Corollary 4) that the positive equilibrium of (7) is 
globally asymptotically stable if $r_{11} \leq 1$ together with other conditions. Further, in [42] it is proved that the positive equilibrium of (7) can also be globally asymptotically stable if $r_{11}>1$. It is known that a system is permanent if its positive equilibrium is globally asymptotically stable [8]. Hence, in $[36,42]$ once again permanence is a consequence but the conditions to ensure it are indirectly obtained.

(b) Our method to obtain the permanence of (7) (or (3)) is different from that in $[35,36,42]$. In particular, the inequalities $m_{1} \leq M_{1}$ and $m_{1}^{*} \leq M_{1}^{*}$ in Definition 1 can be verified here (refer to (A.17), (A.18), (A.26), and (A.27) in Appendix). In most cases if it is proved that each solution of a model is bounded both below and above by some positive constants, then the model is considered to be permanent. However, it is not easy to show the inequalities such as (A.17) and (A.26).

Using Theorem 2, we give the following theorem on the permanence of model (3).

Theorem 4. Suppose that conditions (9) and (10) are satisfied, and

$$
\begin{aligned}
& r_{12}-a_{12}^{(2)} M_{2}>0, \\
& -r_{22}+a_{21}^{(2)} m_{2}>0
\end{aligned}
$$

hold, where

$$
\begin{gathered}
M_{2}=\frac{1}{a_{22}^{(2)}} \exp \left(-r_{22}+a_{21}^{(2)} A_{2}-1\right), \\
A_{2}=\frac{1}{a_{11}^{(2)}} \exp \left(r_{12}-1\right), \\
m_{2}=\frac{r_{12}-a_{12}^{(2)} M_{2}}{a_{11}^{(2)}} \exp \left(r_{12}-a_{12}^{(2)} M_{2}-a_{11}^{(2)} A_{2}\right) .
\end{gathered}
$$

Then, model (3) with initial values (5) is permanent.

3.2. Permanence with Dispersal Corridors. The following theorem gives the sufficient conditions to guarantee the permanence of predator-prey model (4) in patchy environment with dispersal corridors.

Theorem 5. Suppose that

$$
\begin{aligned}
& r_{11}-a_{12}^{(1)} s_{1}>0 \\
& -r_{21}+a_{21}^{(1)} s_{1}>0 \\
& r_{12}-a_{12}^{(2)} s_{2}>0 \\
& -r_{22}+a_{21}^{(2)} s_{2}>0
\end{aligned}
$$

TABLE 1: Sufficient conditions for the permanence of models without/with dispersal corridors.

\begin{tabular}{lcc}
\hline $\begin{array}{l}\text { Concerned with the } \\
\text { permanence }\end{array}$ & $\begin{array}{c}\text { Without dispersal } \\
\text { corridors }\end{array}$ & $\begin{array}{c}\text { With dispersal } \\
\text { corridors }\end{array}$ \\
\hline The prey $x$ in patch 1 & $(9)$ & $(15)$ \\
The predator $y$ in patch 1 & $(10)$ & $(16)$ \\
The prey $x$ in patch 2 & $(12)$ & $(17)$ \\
The predator $y$ in patch 2 & $(13)$ & $(18)$ \\
\hline
\end{tabular}

are satisfied, where

$$
\begin{aligned}
& S_{1}=\frac{1}{a_{22}^{(1)}} \exp \left[-r_{21}+\frac{a_{21}^{(1)}}{a_{11}^{(1)}+d_{12}} \exp \left(r_{11}-1\right)-1\right], \\
& s_{1}=\frac{r_{11}-a_{12}^{(1)} S_{1}}{a_{11}^{(1)}+d_{12}} \exp \left[r_{11}-a_{12}^{(1)} S_{1}-\exp \left(r_{11}-1\right)\right], \\
& S_{2}=\frac{1}{a_{22}^{(2)}} \exp \left[-r_{22}+\frac{a_{21}^{(2)}}{a_{11}^{(2)}+d_{21}} \exp \left(r_{12}-1\right)-1\right], \\
& s_{2}=\frac{r_{12}-a_{12}^{(2)} S_{2}}{a_{11}^{(2)}+d_{21}} \exp \left[r_{12}-a_{12}^{(2)} S_{2}-\exp \left(r_{12}-1\right)\right] .
\end{aligned}
$$

Then, model (4) with initial values (5) is permanent.

Remark 6. Unlike the proof of Theorem 4, in the proof of Theorem 5 (refer to Appendix) the inequalities $m_{1} \leq M_{1}$, $m_{1}^{*} \leq \mathrm{M}_{1}^{*}, m_{2} \leq M_{2}$, and $m_{2}^{*} \leq M_{2}^{*}$ in Definition 1 cannot be verified. This also reinforces Remark 3(b) where we indicate that such inequalities are not easy to prove in most cases.

\section{Effects of Dispersal Corridors}

In Section 3, sufficient conditions for the permanence of models (3) and (4) with initial values (5) are offered in Theorems 4 and 5, respectively. In this section, we will analyze the effects of dispersal corridors on the permanence of discrete predator-prey system by comparing the two sets of sufficient conditions obtained. Recall that the sufficient conditions to guarantee the permanence of predator-prey system without dispersal corridors in patchy environment are (9)(13), while (15)-(18) are the sufficient conditions to guarantee the permanence of the similar system with dispersal corridors. From the proofs of Theorems 4 and 5 (see Appendix), these conditions can be classified in Table 1 .

4.1. Effects of Dispersal Corridors on the Permanence of the Prey. We begin with conditions (9) and (15), which are mainly concerned with the permanence of the prey $x$ in patch 1 under the situation of without or with dispersal corridors. Rewrite (9) as

$$
\begin{aligned}
r_{11} & >a_{12}^{(1)} M_{1} \\
& =\frac{a_{12}^{(1)}}{a_{22}^{(1)}} \exp \left[-r_{21}+\frac{a_{21}^{(1)}}{a_{11}^{(1)}} \exp \left(r_{11}-1\right)-1\right] \triangleq B_{1} .
\end{aligned}
$$


Also, rewrite (15) as

$$
\begin{aligned}
r_{11} & >a_{12}^{(1)} S_{1} \\
& =\frac{a_{12}^{(1)}}{a_{22}^{(1)}} \exp \left[-r_{21}+\frac{a_{21}^{(1)}}{a_{11}^{(1)}+d_{12}} \exp \left(r_{11}-1\right)-1\right] \triangleq B_{2} .
\end{aligned}
$$

On the left sides of (20) and (21), $r_{11}$ is the intrinsic growth rate of the prey $x$ in patch 1 , which makes positive contribution to the population growth of the prey since it is greater than 0 . The right sides of (20) and (21) can be understood as the negative contribution, caused by the intracompetition of species $x$ in patch 1 , the predation by the predator $y$ in patch 1 , and also the dispersion to patch 2 if dispersal corridors exist, to the population growth of the prey. Thus, $\left(r_{11}-B_{1}\right)$ and $\left(r_{11}-B_{2}\right)$ can be interpreted as the net reproduction rates of the prey $x$ in patch 1 without or with dispersal corridors, respectively. Consequently, the biological interpretation of (20) or (21) is that the prey $x$ in patch 1 is permanent if its net reproduction rate is positive.

The only difference between (20) and (21) is whether the dispersal rate $d_{12}$ is involved or not. Recall that $d_{12}(>0)$ is the dispersion rate of the prey $x$ from patch 1 to patch 2 . It is obvious that

$$
B_{1}>B_{2}
$$

or equivalently

$$
r_{11}-B_{1}<r_{11}-B_{2}
$$

This inequality shows that the net reproduction rate of the prey in patch 1 is larger when dispersal corridors are introduced. Hence, the existence of dispersal corridors for the prey $x$ to migrate from patch 1 to patch 2 is helpful to the permanence of the prey in patch 1 . Moreover, if $r_{11}-B_{1}>0$, then $r_{11}-B_{2}>0$ always holds; but if $r_{11}-B_{2}>0$, then we are not certain whether $r_{11}-B_{1}>0$. In other words, with the intrinsic growth rate $r_{11}$ of the prey $x$ in patch 1 keeping the same value regardless of the existence or nonexistence of dispersal corridors, if the prey in patch 1 can be permanent without dispersal corridors, then it is also permanent in patch 1 with dispersal corridors; but if the prey in patch 1 is permanent with dispersal corridors, it may or may not be permanent without the dispersal corridors. In fact, the population growth of the prey in patch 1 is density dependent; hence, an appropriate dispersion from patch 1 to patch 2 may counterbalance the effect of density dependency. Certainly only the dispersion rate $d_{12}$ in an appropriate range is helpful to the permanence of the prey in patch 1 . If the dispersion rate is too large, then the residence of the prey in patch 1 will decrease to a very small number, which will lead to a decrease in the intrinsic growth rate $r_{11}$ and will consequently increase the value of $a_{22}^{(1)}$, the intraspecific competition strength of the predator in patch 1 , due to limited food sources. Hence, in the case of large dispersion rate $d_{12}$, the value of $B_{2}$ will decrease. If the decrease in $r_{11}$ is larger than the decrease in $B_{2}$, then the net reproduction rate of the prey in patch 1 may decrease and (21) may not hold; that is, the permanence of the prey in patch 1 may not be guaranteed.

In summary, an appropriate degree of dispersion of the prey from patch 1 to patch 2, measured by the dispersion rate $d_{12}$, is helpful to the permanence of the prey in patch 1 . Similar conclusion can be drawn in patch 2 by comparing the condition (12) with (17). An appropriate dispersion rate $d_{21}$ of the prey from patch 2 to patch 1 is helpful to the permanence of the prey in patch 2 .

4.2. Effects of Dispersal Corridors on the Permanence of the Predator. We begin with conditions (10) and (16), which are mainly concerned with the permanence of the predator $y$ in patch 1 under the situation of without or with dispersal corridors. Rewrite (10) as

$$
\begin{aligned}
r_{21}< & a_{21}^{(1)} m_{1} \\
= & a_{21}^{(1)} \frac{r_{11}-a_{12}^{(1)} M_{1}}{a_{11}^{(1)}} \\
& \times \exp \left[r_{11}-a_{12}^{(1)} M_{1}-\exp \left(r_{11}-1\right)\right] \triangleq B_{3},
\end{aligned}
$$

and rewrite (16) as

$$
\begin{aligned}
r_{21}< & a_{21}^{(1)} s_{1} \\
= & a_{21}^{(1)} \frac{r_{11}-a_{12}^{(1)} S_{1}}{a_{11}^{(1)}+d_{12}} \\
& \quad \times \exp \left[r_{11}-a_{12}^{(1)} S_{1}-\exp \left(r_{11}-1\right)\right] \triangleq B_{4} .
\end{aligned}
$$

For convenience, we also list the expressions of $M_{1}$ and $S_{1}$ (refer to Theorems 2 and 5) as follows:

$$
\begin{gathered}
M_{1}=\frac{1}{a_{22}^{(1)}} \exp \left[-r_{21}+\frac{a_{21}^{(1)}}{a_{11}^{(1)}} \exp \left(r_{11}-1\right)-1\right], \\
S_{1}=\frac{1}{a_{22}^{(1)}} \exp \left[-r_{21}+\frac{a_{21}^{(1)}}{a_{11}^{(1)}+d_{12}} \exp \left(r_{11}-1\right)-1\right] .
\end{gathered}
$$

On the left sides of (24) and (25), $r_{21}$ is the death rate of the predator $y$ in patch 1 . The right sides of (24) and (25) can be understood as the positive contribution to the population growth of the predator in patch 1 due to the predation. Hence, $\left(B_{3}-r_{21}\right)$ and $\left(B_{4}-r_{21}\right)$ can be interpreted as the net reproduction rates of the predator $y$ in patch 1 without or with dispersal corridors, respectively. Consequently, the biological interpretation of (24) or (25) is that the predator $y$ in patch 1 is permanent if its net reproduction rate is positive.

There are two "places" in (25) where the dispersal rate $d_{12}$ appears; one is in the denominator of $B_{4}$, while another is in the term $S_{1}$. We will discuss the biological implications of them. First, it is clear that the $d_{12}$ that is explicit in the denominator of (25) has a diminishing effect on $B_{4}$. Therefore, this dispersal rate in the denominator is disadvantageous to the net reproduction rate of the population growth, further the permanence, of the predator in patch 1 . In fact, the migration 
of the prey from patch 1 to patch 2, while the predator remains fixed to patch 1 , causes the decrease in the food sources of the predator, which in turn is disadvantageous to the permanence of the predator in patch 1 .

Next, we consider the effect of the dispersal rate $d_{12}$ in the term $S_{1}$. It is obvious from (26) that $S_{1}<M_{1}$; thus

$$
r_{11}-a_{12}^{(1)} S_{1}>r_{11}-a_{12}^{(1)} M_{1}
$$

or equivalently (see (20) and (21))

$$
r_{11}-B_{2}>r_{11}-B_{1}
$$

which is exactly (23). We have observed earlier that the net reproduction rate of the prey in patch 1 with dispersal corridors is larger than that without dispersal corridors. In the following, we continue to analyze how this larger net reproduction rate of the prey in patch 1 impacts the net reproduction rate of the predator in patch 1 .

Consider the function $g(x)=x \exp (x-a)$ where $a>$ 0 in the interval $(0,+\infty)$. The derivative of $g(x)$ is $g^{\prime}(x)=$ $(1+x) \exp (x-a)>0$, so $g(x)$ is monotonically increasing in $(0,+\infty)$. Thus, noting (27) we have

$$
\begin{aligned}
\left(r_{11}-\right. & \left.a_{12}^{(1)} S_{1}\right) \exp \left[r_{11}-a_{12}^{(1)} S_{1}-\exp \left(r_{11}-1\right)\right] \\
& >\left(r_{11}-a_{12}^{(1)} M_{1}\right) \exp \left[r_{11}-a_{12}^{(1)} M_{1}-\exp \left(r_{11}-1\right)\right]
\end{aligned}
$$

Noting the definitions of $B_{3}$ and $B_{4}$, inequality (29) shows that the dispersal rate $d_{12}$ which appeared in $S_{1}$ is advantageous to the increase of the net reproduction rate of the predator in patch 1, which in turn is helpful to the permanence of the predator in patch 1 . In fact, from (27) (or (23)) this dispersion rate in $S_{1}$ increases the net reproduction rate of the prey in patch 1 , which consequently increases the food supply for the predator in patch 1 . As a result, this contributes positively to the net reproduction rate of the predator in patch 1 , which further helps to sustain the permanence of the predator in patch 1 .

In summary, the effects of the dispersion of the prey from patch 1 to patch 2 , measured by the dispersal rate $d_{12}$, on the permanence of the predator in patch 1 are twofold: one is disadvantageous-contributed by the presence of $d_{12}$ in the denominator of $B_{4}$, while the other is advantageouscontributed by the presence of $d_{12}$ in $S_{1}$. Similar conclusion can be drawn in patch 2 by comparing the condition (13) with (18). The dispersion of the prey from patch 2 to patch 1 , measured by the dispersal rate $d_{21}$, has multiple effects, both advantageous and disadvantageous, on the permanence of the predator in patch 2 .

Remark 7. From the two sets of permanence conditions in Theorems 4 and 5, no conclusion can be drawn on the effects of $d_{21}$ on the permanence of the prey and the predator in patch 1; also the effects of $d_{12}$ on the permanence of the prey and the predator in patch 2 are unknown.

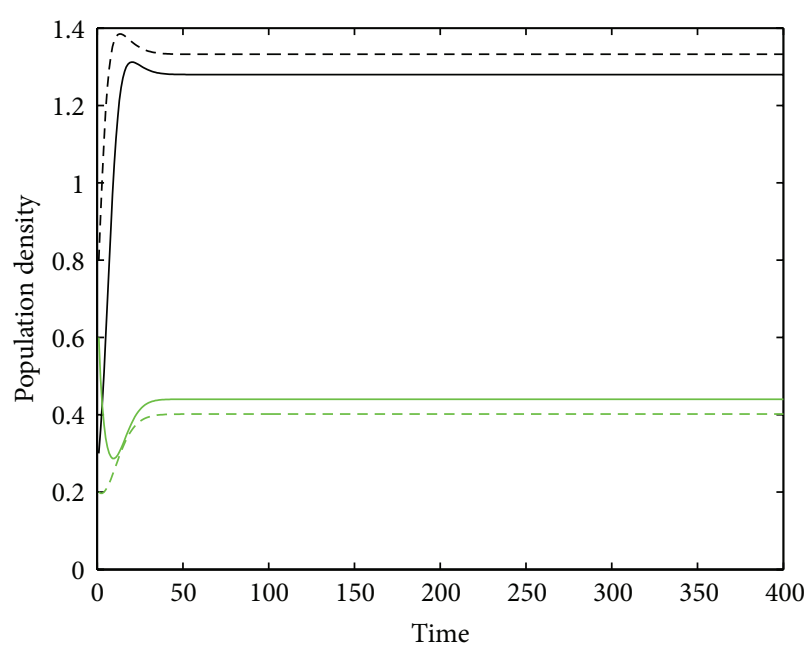

FIGURE 1: Permanence of model (30). The black solid (dotted) line is the population variation of the prey in patch 1 (patch 2). The green solid (dotted) line is the population variation of the predator in patch 1 (patch 2).

\section{Numerical Simulation}

In this section, we present five numerical examples to confirm the theoretical results obtained earlier as well as to illustrate the effects of the dispersal corridors. All the examples are processed by MATLAB. The first example below confirms the correctness of Theorem 4 .

Example 8. Consider the following predator-prey model (3) in patchy environment without dispersal corridors:

$$
\begin{aligned}
& x_{1}(n+1)=x_{1}(n) \exp \left[0.3-0.2 x_{1}(n)-0.1 y_{1}(n)\right], \\
& y_{1}(n+1)=y_{1}(n) \exp \left[-0.06+0.15 x_{1}(n)-0.3 y_{1}(n)\right], \\
& x_{2}(n+1)=x_{2}(n) \exp \left[0.32-0.21 x_{2}(n)-0.1 y_{2}(n)\right], \\
& y_{2}(n+1)=y_{2}(n) \exp \left[-0.07+0.14 x_{2}(n)-0.29 y_{2}(n)\right] .
\end{aligned}
$$

Direct computation gives

$$
\begin{array}{ll}
r_{11}-a_{12}^{(1)} M_{1}=0.1324>0, & -r_{21}+a_{21}^{(1)} m_{1}=0.0090>0, \\
r_{12}-a_{12}^{(2)} M_{2}=0.1524>0, & -r_{22}+a_{21}^{(2)} m_{2}=0.0023>0 .
\end{array}
$$

Hence, the conditions of Theorem 4 are all satisfied. By Theorem 4, model (30) is permanent and this is illustrated in Figure 1.

The next example illustrates Theorem 5. 
Example 9. Consider the following predator-prey model (4) in patchy environment with dispersal corridors:

$$
\begin{aligned}
& x_{1}(n+1) \\
& =x_{1}(n) \exp \left[0.3-0.05 x_{1}(n)-0.1 y_{1}(n)\right. \\
& \left.-0.1 x_{1}(n)+0.15 x_{2}(n)\right], \\
& y_{1}(n+1) \\
& =y_{1}(n) \exp \left[-0.07+0.15 x_{1}(n)-0.3 y_{1}(n)\right. \\
& \left.+0.15 * 0.15 x_{2}(n)\right], \\
& x_{2}(n+1) \\
& =x_{2}(n) \exp \left[0.52-0.35 x_{2}(n)-0.15 y_{2}(n)\right. \\
& \left.+0.1 x_{1}(n)-0.15 x_{2}(n)\right], \\
& y_{2}(n+1) \\
& =y_{2}(n) \exp \left[-0.08+0.3 x_{2}(n)-0.25 y_{2}(n)\right. \\
& \left.+0.3 * 0.1 x_{1}(n)\right] \text {. }
\end{aligned}
$$

In model (32), the dispersal rates are $d_{12}=0.1$ and $d_{21}=$ 0.15 . Also, we set $\alpha=a_{21}^{(1)}=0.15$ and $\beta=a_{21}^{(2)}=0.3$; that is, we assume that the predation rate of the predator on the prey which migrated from another patch is the same as the predation rate on the prey originally in the patch. By direct computation, we have all the conditions of Theorem 5 satisfied as follows:

$$
\begin{array}{ll}
r_{11}-a_{12}^{(1)} S_{1}=0.1121>0, & -r_{21}+a_{21}^{(1)} s_{1}=0.0063>0, \\
r_{12}-a_{12}^{(2)} S_{2}=0.2246>0, & -r_{22}+a_{21}^{(2)} s_{2}=0.0109>0 .
\end{array}
$$

Hence, by Theorem 5 model (32) is permanent. Figure 2 illustrates the permanence of model (32).

Next, we present an example to show that dispersal corridors may be disadvantageous to the permanence of the ecosystem.

Example 10. Consider the following two models:

$$
\begin{gathered}
x_{1}(n+1)=x_{1}(n) \exp \left[0.29-0.2 x_{1}(n)-0.1 y_{1}(n)\right], \\
y_{1}(n+1)=y_{1}(n) \exp \left[-0.06+0.15 x_{1}(n)-0.3 y_{1}(n)\right], \\
x_{2}(n+1)=x_{2}(n) \exp \left[0.32-0.21 x_{2}(n)-0.1 y_{2}(n)\right], \\
y_{2}(n+1)=y_{2}(n) \exp \left[-0.07+0.14 x_{2}(n)-0.29 y_{2}(n)\right], \\
x_{1}(n+1)=x_{1}(n) \exp \left[0.29-0.2 x_{1}(n)-0.1 y_{1}(n)\right. \\
\left.\quad-0.61 x_{1}(n)\right], \\
y_{1}(n+1)=y_{1}(n) \exp \left[-0.06+0.15 x_{1}(n)-0.3 y_{1}(n)\right],
\end{gathered}
$$

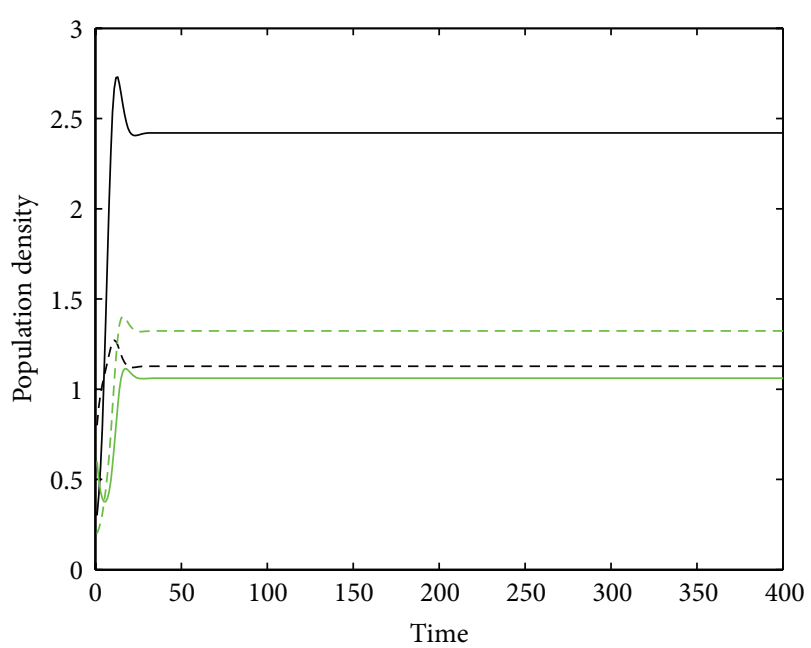

Figure 2: Permanence of model (32). The black solid (dotted) line is the population variation of the prey in patch 1 (patch 2). The green solid (dotted) line is the population variation of the predator in patch 1 (patch 2).

$$
\begin{aligned}
x_{2}(n+1)=x_{2}(n) \exp [ & 0.32-0.21 x_{2}(n)-0.1 y_{2}(n) \\
+ & \left.0.61 x_{1}(n)\right], \\
y_{2}(n+1)=y_{2}(n) \exp [ & -0.07+0.14 x_{2}(n)-0.29 y_{2}(n) \\
+ & \left.0.14 * 0.61 x_{1}(n)\right] .
\end{aligned}
$$

The difference between (34) and (35) is only whether dispersal corridors exist or not; all other coefficients are the same. Model (34) is without dispersal corridors, and a direct computation shows that all the conditions of Theorem 4 are satisfied as follows:

$$
\begin{array}{ll}
r_{11}-a_{12}^{(1)} M_{1}=0.1230>0, & -r_{21}+a_{21}^{(1)} m_{1}=0.0038>0, \\
r_{12}-a_{12}^{(2)} M_{2}=0.1542>0, & -r_{22}+a_{21}^{(2)} m_{2}=0.0023>0 .
\end{array}
$$

Hence, by Theorem 4 model (34) is permanent. Figure 3(a) illustrates the permanence of model (34).

Model (35) has dispersal corridors with $d_{12}=0.61, d_{21}=$ 0 , and $\beta=a_{21}^{(2)}=0.14$. Checking the conditions of Theorem 5 , we find

$$
\begin{array}{cl}
r_{11}-a_{12}^{(1)} S_{1}=0.1635>0, & -r_{21}+a_{21}^{(1)} s_{1}=-0.0382<0, \\
r_{12}-a_{12}^{(2)} S_{2}=0.1542>0, & -r_{22}+a_{21}^{(2)} s_{2}=0.0023>0 .
\end{array}
$$

Hence, not all the conditions of Theorem 5 are satisfied and we cannot ensure the permanence of model (35). In fact, by numerical simulation we find that the predator in patch 1 is driven to extinction and so model (35) is nonpermanent. This is depicted in Figure 3(b). 


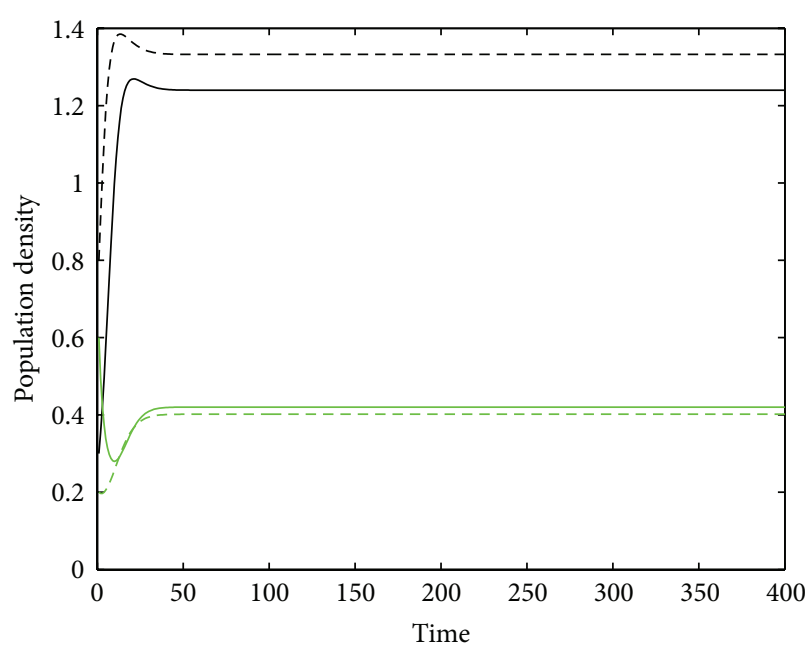

(a)

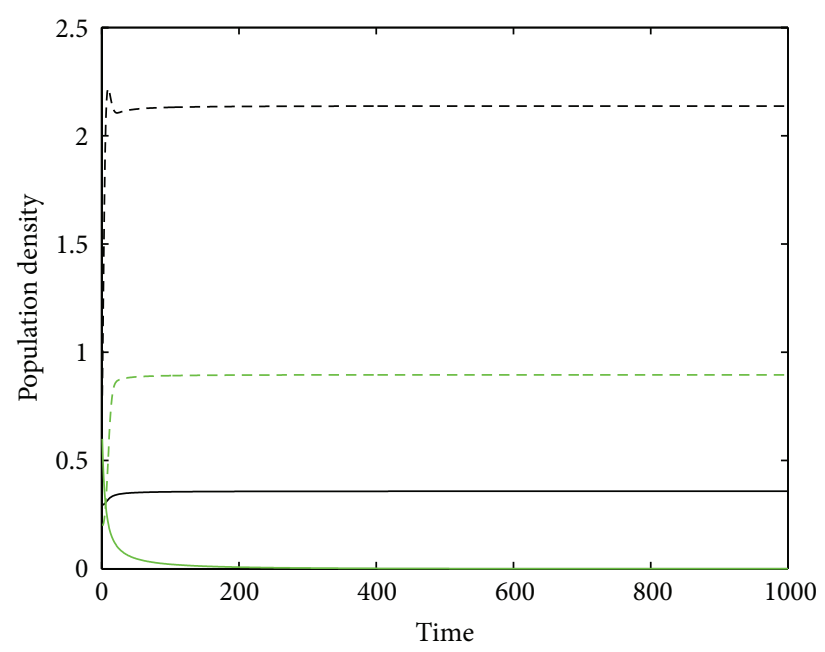

(b)

FIgURE 3: The dynamics of models (34) and (35). (a) is the dynamics of (34). It is observed that model (34) is permanent. (b) is the dynamics of (35). It is observed that the predator in patch 1 is driven to extinction when dispersal corridors are introduced. The black solid (dotted) line is the population variation of the prey in patch 1 (patch 2). The green solid (dotted) line is the population variation of the predator in patch 1 (patch 2).

This example shows that the dispersion of the prey from patch 1 to patch 2 by the dispersal corridors may be disadvantageous to the predator in patch 1 , which has been discussed in Section 4.2. Further, it is observed from the above calculations that the introduction of $d_{12}$ increases the net reproduction rate $\left(r_{11}-a_{12}^{(1)} S_{1}\right)$ of the prey in patch 1 from 0.1230 to 0.1635 , which has been noted in Section 4.1.

Next, we give an example to show that dispersal corridors may be helpful to the permanence of the ecosystem.

Example 11. Consider the following two models:

$$
\begin{gathered}
x_{1}(n+1)=x_{1}(n) \exp \left[0.05-0.5 x_{1}(n)-0.16 y_{1}(n)\right], \\
y_{1}(n+1)=y_{1}(n) \exp \left[-0.02+0.2 x_{1}(n)-0.5 y_{1}(n)\right], \\
x_{2}(n+1)=x_{2}(n) \exp \left[0.52-0.35 x_{2}(n)-0.15 y_{2}(n)\right], \\
y_{2}(n+1)=y_{2}(n) \exp \left[-0.08+0.3 x_{2}(n)-0.25 y_{2}(n)\right], \\
x_{1}(n+1)=x_{1}(n) \exp \left[0.05-0.5 x_{1}(n)-0.16 y_{1}(n)\right. \\
\left.\quad-d_{12} x_{1}(n)+d_{21} x_{2}(n)\right], \\
y_{1}(n+1)=y_{1}(n) \exp \left[-0.02+0.2 x_{1}(n)-0.5 y_{1}(n)\right. \\
\left.\quad+0.2 * d_{21} x_{2}(n)\right], \\
x_{2}(n+1)=x_{2}(n) \exp \left[0.52-0.35 x_{2}(n)-0.15 y_{2}(n)\right. \\
\left.+d_{12} x_{1}(n)-d_{21} x_{2}(n)\right], \\
y_{2}(n+1)=y_{2}(n) \exp \left[-0.08+0.3 x_{2}(n)-0.25 y_{2}(n)\right. \\
\left.+0.3 * d_{12} x_{1}(n)\right] .
\end{gathered}
$$

The difference between (38) and (39) is only whether dispersal corridors exist or not; all other coefficients are the same. Model (38) is without dispersal corridors, and a direct computation yields

$$
\begin{aligned}
& r_{11}-a_{12}^{(1)} M_{1}=-0.0847<0, \quad-r_{21}+a_{21}^{(1)} m_{1}=-0.0411<0, \\
& r_{12}-a_{12}^{(2)} M_{2}=0.1737>0, \quad-r_{22}+a_{21}^{(2)} m_{2}=0.0154>0 .
\end{aligned}
$$

Hence, not all the conditions of Theorem 4 are satisfied and we cannot guarantee the permanence of (38). Indeed, by numerical simulation it is found that the predator in patch 1 will be driven to extinction. So model (38) is nonpermanent and this is illustrated in Figure 4(a).

Model (39) has dispersal corridors with $d_{12}>0, d_{21}>0$, $\alpha=a_{21}^{(1)}=0.2$, and $\beta=a_{21}^{(2)}=0.3$. Suppose

$$
d_{12}=0.55, \quad d_{21}=0.21 \text {. }
$$

By numerical simulation we find that the predator in patch 1 is now permanent, and model (39), as a whole, is permanent. This is depicted in Figure 4(b). Note, however, that model (39) does not satisfy some of the conditions of Theorem 5 , as

$$
\begin{array}{cl}
r_{11}-a_{12}^{(1)} S_{1}=-0.0742<0, & -r_{21}+a_{21}^{(1)} s_{1}=-0.0289<0, \\
r_{12}-a_{12}^{(2)} S_{2}=0.2362>0, & -r_{22}+a_{21}^{(2)} s_{2}=0.0063>0 .
\end{array}
$$

This simply reinforces the fact that the conditions in Theorem 5 are sufficient conditions.

We observe that the introduction of dispersal corridors leads to (i) an increase in the net reproduction rates of both 


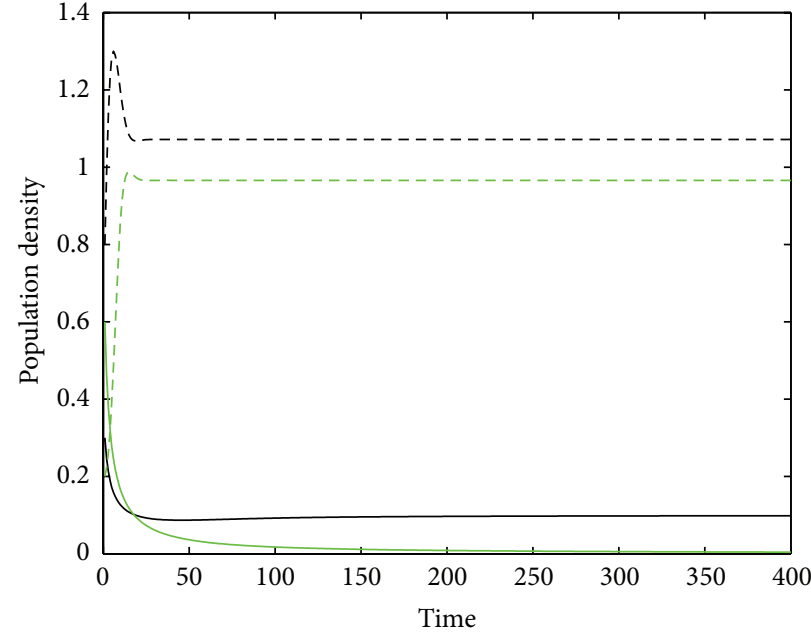

(a)

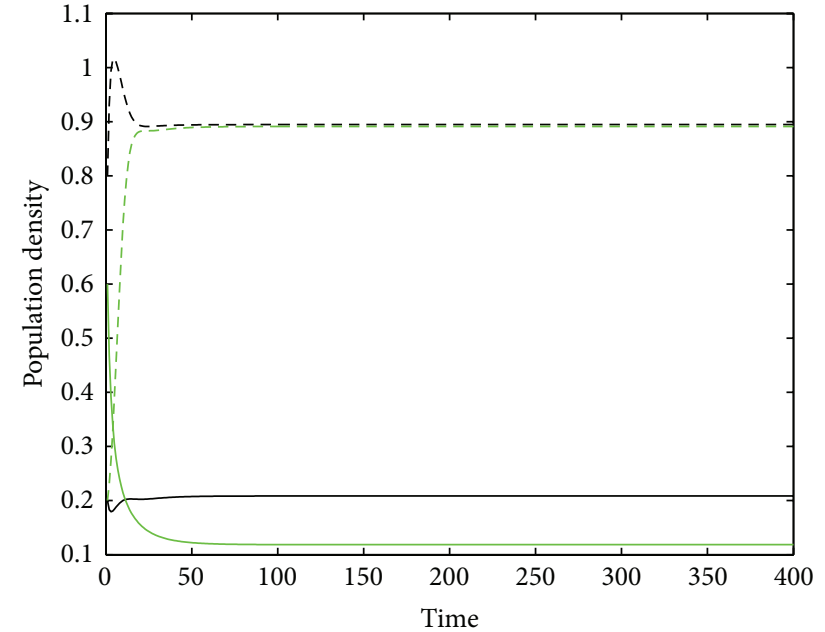

(b)

FIGURE 4: The dynamics of models (38) and (39) subject to (41). (a) is the dynamics of (38). It is observed that the predator in patch 1 will go to extinction. (b) is the dynamics of (39). It is observed that the predator in patch 1 is permanent now due to the dispersal corridors, and (39), as a whole, is permanent. The black solid (dotted) line is the population variation of the prey in patch 1 (patch 2). The green solid (dotted) line is the population variation of the predator in patch 1 (patch 2).

TABLE 2: Net reproduction rates and the permanence of model (39) under different dispersal rates.

\begin{tabular}{|c|c|c|c|c|c|}
\hline Dispersal rates & $R_{1}^{x}$ & $R_{1}^{y}$ & $R_{2}^{x}$ & $R_{2}^{y}$ & Permanence of model (39) \\
\hline$d_{12}=0, d_{21}=0$ & -0.0847 & -0.0411 & 0.1737 & 0.0154 & No, predator in patch 1 is extinct \\
\hline$d_{12}=0.18, d_{21}=0$ & -0.0793 & -0.0346 & 0.1737 & 0.0154 & No, predator in patch 1 is extinct \\
\hline$d_{12}=0.36, d_{21}=0$ & -0.0762 & -0.0312 & 0.1737 & 0.0154 & No, predator in patch 1 is extinct \\
\hline$d_{12}=0.55, d_{21}=0$ & -0.0742 & -0.0289 & 0.1737 & 0.0154 & No, predator in patch 1 is extinct \\
\hline$d_{12}=1, d_{21}=0$ & -0.0715 & -0.0260 & 0.1737 & 0.0154 & No, predator in patch 1 is extinct \\
\hline$d_{12}=0.55, d_{21}=0.02$ & -0.0742 & -0.0289 & 0.1835 & 0.0163 & No, predator in patch 1 is extinct \\
\hline$d_{12}=0.55, d_{21}=0.07$ & -0.0742 & -0.0289 & 0.2030 & 0.0157 & Yes \\
\hline$d_{12}=0.55, d_{21}=0.14$ & -0.0742 & -0.0289 & 0.2224 & 0.0116 & Yes \\
\hline$d_{12}=0.55, d_{21}=0.21$ & -0.0742 & -0.0289 & 0.2362 & 0.0063 & Yes \\
\hline$d_{12}=0.55, d_{21}=0.28$ & -0.0742 & -0.0289 & 0.2464 & 0.00086 & Yes \\
\hline
\end{tabular}

the prey and the predator in patch 1 as well as the prey in patch 2 and (ii) a decrease in the net reproduction rate of the predator in patch 2 . The overall effect on the permanence of model (39) is positive. This example shows that the introduction of dispersal corridors to a patchy ecosystem may be advantageous to the permanence of the ecosystem.

We will further investigate the effects of dispersal corridors on model (39). For this, in Table 2, for different values of $d_{12}$ and $d_{21}$ we list the net reproduction rate $R_{i}^{x}$ of the prey $x$ in patch $i$, the net reproduction rate $R_{i}^{y}$ of the predator $y$ in patch $i, i \in\{1,2\}$, and the permanence status of model (39). Recall that, for a model with dispersal corridors,

$$
\begin{array}{ll}
R_{1}^{x}=r_{11}-a_{12}^{(1)} S_{1}, & R_{1}^{y}=-r_{21}+a_{21}^{(1)} s_{1}, \\
R_{2}^{x}=r_{12}-a_{12}^{(2)} S_{2}, & R_{2}^{y}=-r_{22}+a_{21}^{(2)} s_{2} .
\end{array}
$$

From Table 2, we observe the following.

(a) The effect of dispersal corridors on the net reproduction rate of prey is positive; specifically, $R_{1}^{x}$ increases as $d_{12}$ increases and $R_{2}^{x}$ increases as $d_{21}$ increases. This coincides with the discussion in Section 4.1.

(b) The effects of dispersal corridors on the net reproduction rate of predator are twofold: advantageous and disadvantageous. For a fixed $d_{12}=0.55, R_{2}^{y}$ first increases and then decreases as the value of $d_{21}$ increases from 0 to 0.28 .

(c) The entire ecosystem cannot be permanent by the sole effect of $d_{12}$ (i.e., $d_{21}=0$ ). If the value of $d_{12}$ increases to sufficiently large, even the prey in patch 1 will undergo extinction due to unpredicted environmental changes, as the population density of the prey in patch 1 will become very small. If the prey in patch 1 becomes extinct, so will the predator in patch 1 , since there are no food sources.

(d) Noting (c) above, we further observe that the ecosystem is also nonpermanent if $d_{21}$ is too small; an appropriate value of $d_{21}$ is needed to induce a permanent (39). 
So far equilibrium dynamics have been observed in Examples 8-11. Since it is well known that Ricker type model has complex dynamics, we give an example with more complex dynamics to illustrate our results.

Example 12. Consider the following two models:

$$
\begin{gathered}
x_{1}(n+1)=x_{1}(n) \exp \left[2.4-0.2 x_{1}(n)-0.1 y_{1}(n)\right], \\
y_{1}(n+1)=y_{1}(n) \exp \left[-0.1+0.15 x_{1}(n)-0.3 y_{1}(n)\right], \\
x_{2}(n+1)=x_{2}(n) \exp \left[0.4-0.21 x_{2}(n)-0.1 y_{2}(n)\right], \\
y_{2}(n+1)=y_{2}(n) \exp \left[-0.07+0.14 x_{2}(n)-0.69 y_{2}(n)\right] \\
\left.\quad+0.6 x_{2}(n)\right] \\
x_{1}(n+1)=x_{1}(n) \exp \left[2.4-0.2 x_{1}(n)-0.1 y_{1}(n)\right. \\
y_{1}(n+1)=y_{1}(n) \exp \left[-0.1+0.15 x_{1}(n)-0.3 y_{1}(n)\right. \\
\left.+0.15 * 0.6 x_{2}(n)\right], \\
x_{2}(n+1)=x_{2}(n) \exp \left[0.4-0.21 x_{2}(n)-0.1 y_{2}(n)\right. \\
\left.\quad-0.6 x_{2}(n)\right], \\
y_{2}(n+1)=y_{2}(n) \exp \left[-0.07+0.14 x_{2}(n)-0.69 y_{2}(n)\right] .
\end{gathered}
$$

The difference between (44) and (45) is only whether dispersal corridors exist or not; all other coefficients are the same. Model (44) is without dispersal corridors, and a direct computation yields

$$
\begin{aligned}
& r_{11}-a_{12}^{(1)} M_{1}=0.0772>0, \quad-r_{21}+a_{21}^{(1)} m_{1}=-0.0989<0, \\
& r_{12}-a_{12}^{(2)} M_{2}=0.3283>0, \quad-r_{22}+a_{21}^{(2)} m_{2}=0.1056>0 .
\end{aligned}
$$

Though not all the conditions of Theorem 4 are satisfied, model (44) is permanent which is illustrated in Figure 5(a). It is observed from Figure 5(a) that the subsystem of patch 2 has a stable positive equilibrium while the subsystem of patch 1 does not have such stable equilibrium though it is permanent. Further illustration of this subsystem of patch 1 is given in Figure 5(b). Figure 5(b) is the phase diagram of $\left(x_{1}, y_{1}\right)$ and it is observed that the subsystem of patch 1 of model (44) has a periodic solution of period 3. In fact, the periodic solution is $\left(x_{1}, y_{1}\right)=(18.7232,1.1061),(4.3689,11.9115)$, $(6.1079,0.5824)$.

Model (45) has dispersal corridors with $d_{21}=0.6, d_{12}=$ 0 , and $\alpha=a_{21}^{(1)}=0.15$. The net reproduction rates are computed as follows:

$$
\begin{array}{ll}
r_{11}-a_{12}^{(1)} S_{1}=0.0772>0, & -r_{21}+a_{21}^{(1)} s_{1}=-0.0989<0, \\
r_{12}-a_{12}^{(2)} S_{2}=0.3453>0, & -r_{22}+a_{21}^{(2)} s_{2}=-0.0213<0 .
\end{array}
$$

The dynamics of (45) are presented in Figure 6(a). It is observed that the predator in patch 2 will become extinct and consequently model (45) is not permanent. Figure 6(b) illustrates that the periodic solution observed in the subsystem of patch 1 of model (44) now vanishes due to the dispersion.

Comparing (46) and (47), we observe that when $d_{21}>$ 0 , (i) the net reproduction rate of the prey in patch 2 increases, which has been noted in Section 4.1 and (ii) the net reproduction rate of the predator in patch 2 decreases its value from positive to negative; this shows that the dispersion of the prey from patch 2 to patch 1 has an adverse effect on the permanence of the predator in patch 2, which has been discussed in Section 4.2. This example shows that large dispersal rates of the prey may destroy the permanence of a predator-prey system.

\section{Conclusion}

The paper is mainly involved with the effects of dispersal corridors on the permanence of discrete predator-prey models in patchy environment. We have introduced two models of Ricker type undergoing the effect of density dependencymodel (3) is in patchy environment without dispersal corridors and model (4) is with dispersal corridors. Two sets of sufficient conditions to guarantee the permanence of the two models are then obtained through theoretical studies, respectively. In Theorem 4, conditions (9)-(13) guarantee the permanence of model (3); this result is illustrated by Example 8. In Theorem 5, conditions (15)-(18) ensure the permanence of model (4); this result is also numerically illustrated by Example 9 .

Through mathematical analysis of the two sets of sufficient conditions, combined with the actual biological background of models (3) and (4), we have discussed the effects of dispersal corridors on the permanence of discrete predatorprey model. It is found that the existence of dispersal corridors for the prey to migrate, such as from patch $i$ to patch $j$, while the predator remains fixed to the original patch, is helpful to the permanence of the prey in patch $i$ under density dependent circumstances due to an increased net reproduction rate of the prey in patch $i$. Moreover, it is also found that the migration of prey, such as from patch $i$ to patch $j$, is both advantageous and disadvantageous to the predator in patch $i$ because the dispersal rate $d_{i j}$ has both positive and negative effects on the net reproduction rate of the predator in patch $i$. Examples 10-12 are presented to illustrate the positive/negative effects of the dispersal corridors.

\section{Appendix}

In this section, we present the proofs of Theorems 2, 4, and 5. First we state a lemma which is useful in proving the theorems; this lemma is the autonomous case of Lemma 2 in [43].

Lemma 13. Suppose $\{z(k)\}$ satisfies

$$
z(k+1) \geq z(k) \exp [r-a z(k)],
$$

for $k \geq K$, where $a$ and $r$ are positive constants, $K$ is a positive integer, and $z(K)>0$. Further, assume that 

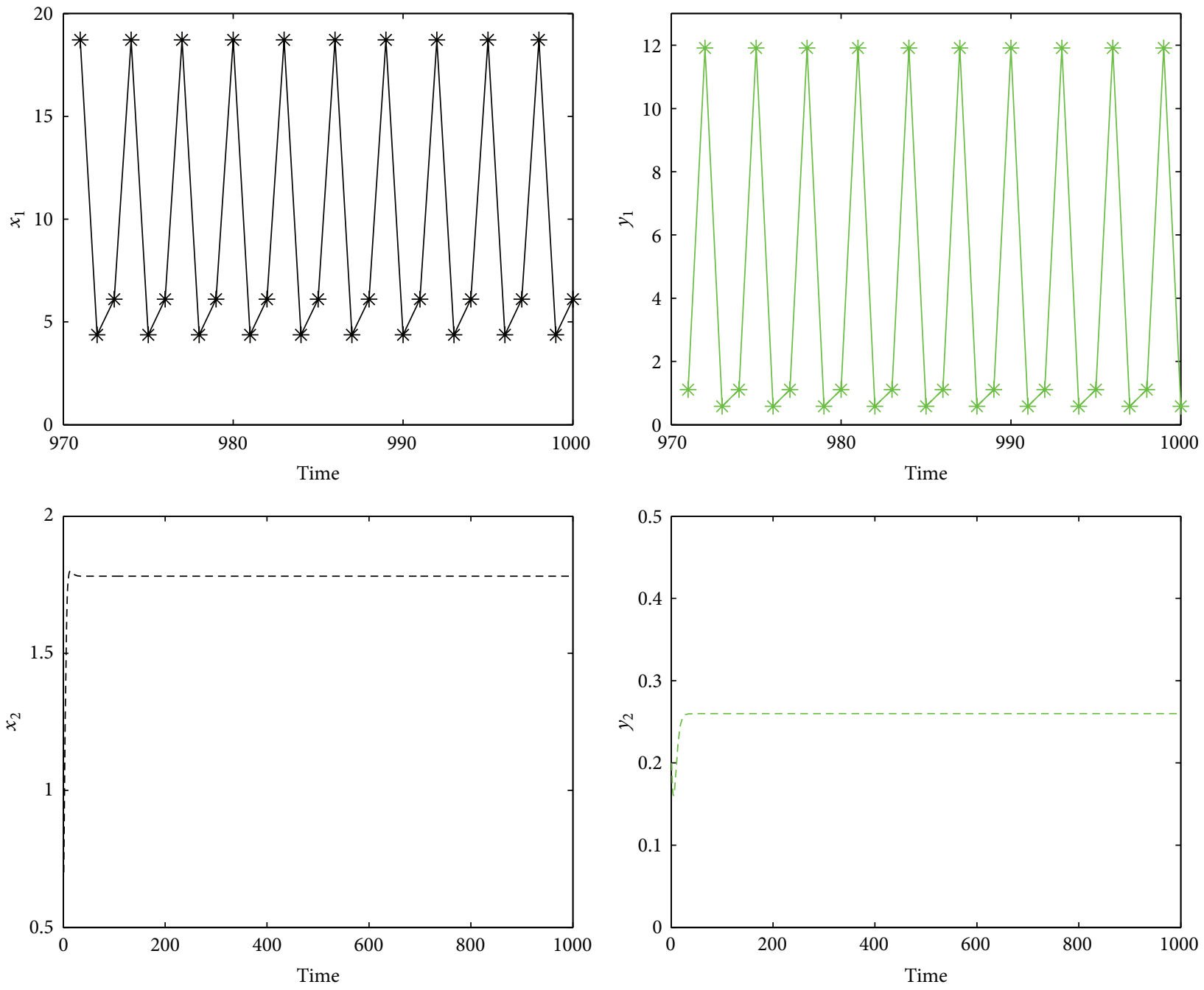

(a)

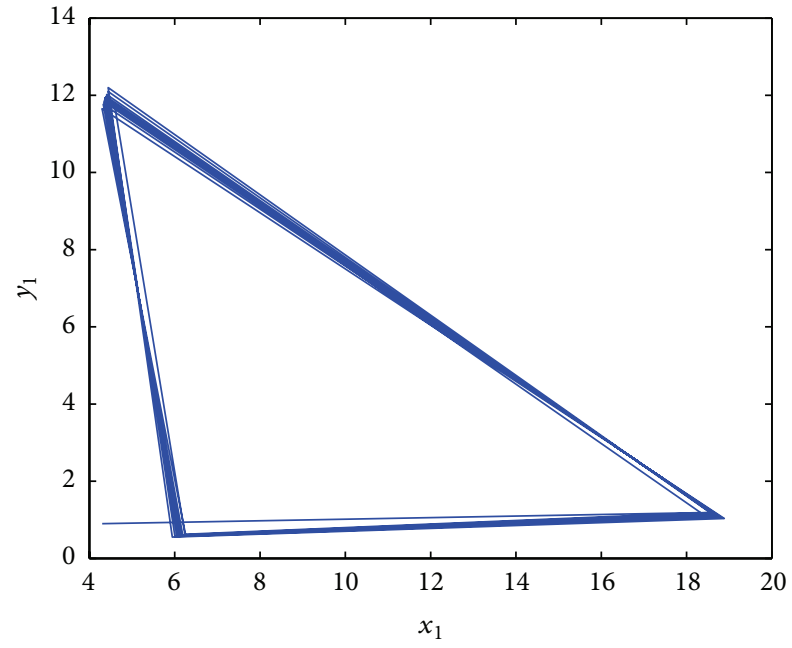

(b)

Figure 5: The dynamics of model (44). (a) is the dynamics of (44). It is observed that model (44) is permanent. The subsystem of patch 2 has a stable positive equilibrium, while oscillating population densities for both the prey and the predator are observed in the subsystem of patch 1. (b) is the dynamics of the subsystem of patch 1 illustrated in the phase diagram $\left(x_{1}, y_{1}\right)$. A periodic solution of period 3 is observed. 

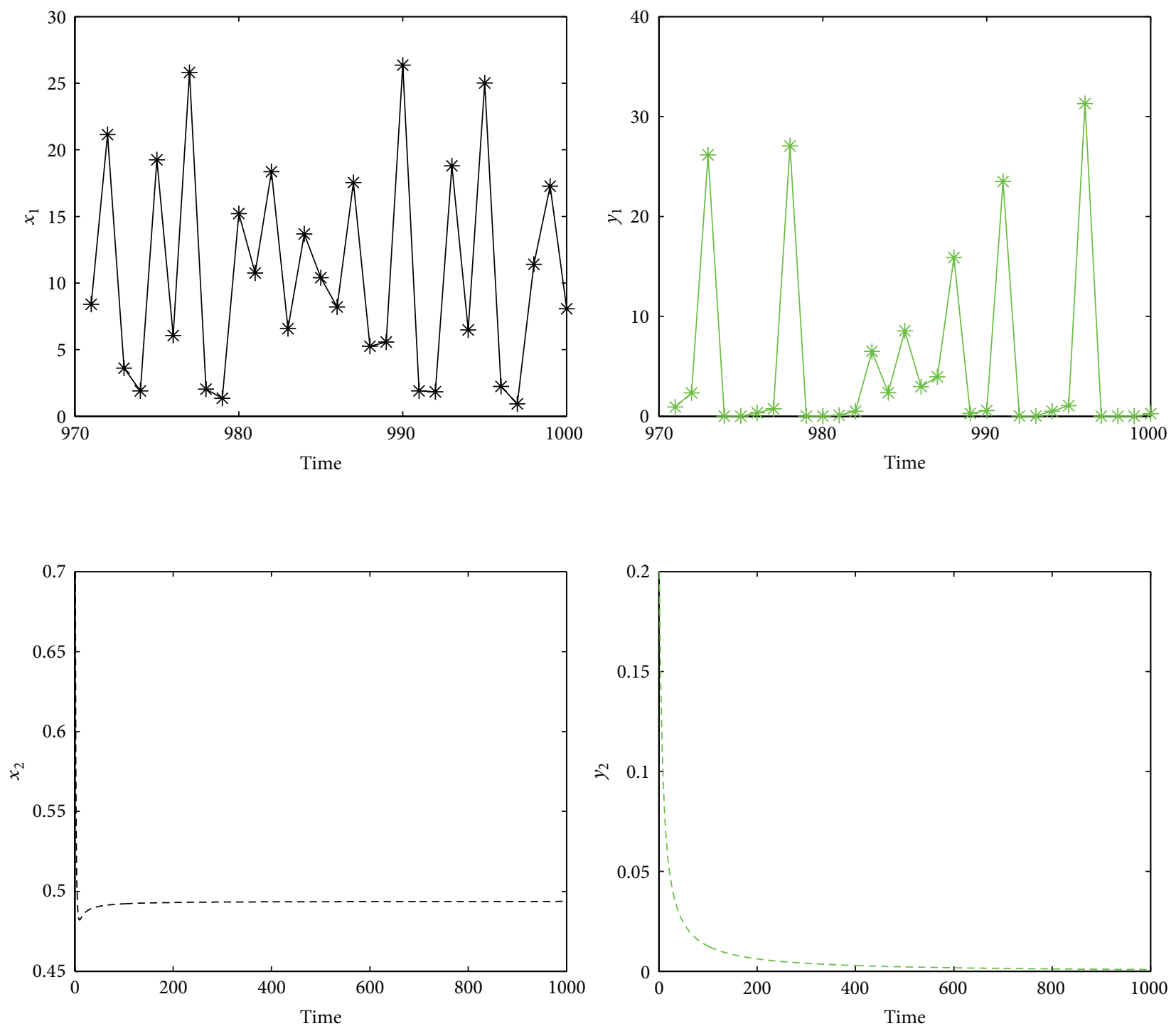

(a)

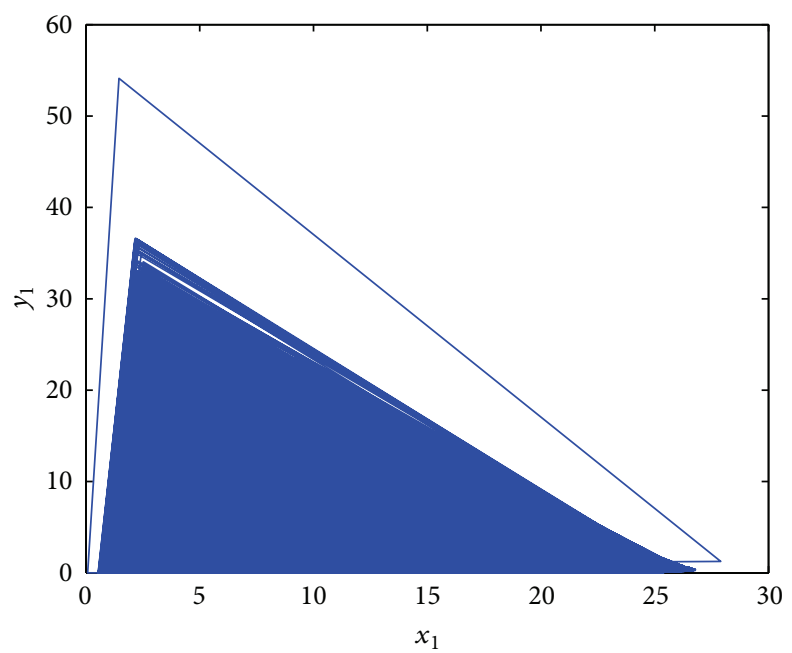

(b)

Figure 6: The dynamics of model (45). (a) is the dynamics of (45). It is observed that the predator in patch 2 will become extinct due to the dispersal corridors, and (45), as a whole, is not permanent. (b) is the dynamics of the subsystem of patch 1 illustrated in the phase diagram $\left(x_{1}, y_{1}\right)$. The periodic solution in Figure 5(b) vanishes due to dispersion. 
$\lim \sup _{k \rightarrow+\infty} z(k) \leq A$ and $a A / r>1$, where $A$ is a positive constant. Then,

$$
\liminf _{k \rightarrow+\infty} z(k) \geq \frac{r}{a} \exp (r-a A) .
$$

Proof of Theorem 2. We will first establish the first inequality in Definition 1. To begin, from the first equation of (7) we have

$$
x_{1}(n+1) \leq x_{1}(n) \exp \left(r_{11}-a_{11}^{(1)} x_{1}(n)\right) .
$$

It is straightforward that the maximum of the function $z(x)=$ $x \exp (r-a x)$ where $a>0$ in the interval $[0,+\infty)$ is $z_{\max }=$ $(1 / a) \exp (r-1)$. Therefore, it follows from (A.3) that

$$
\limsup _{n \rightarrow+\infty} x_{1}(n) \leq \frac{1}{a_{11}^{(1)}} \exp \left(r_{11}-1\right) \triangleq A_{1} .
$$

Let $\epsilon>0$ be arbitrarily given. From (A.4), there exists a positive integer $N_{1}$ such that

$$
x_{1}(n) \leq A_{1}+\epsilon, \quad n>N_{1} .
$$

Using (A.5) in the second equation of (7) gives

$$
\begin{array}{r}
y_{1}(n+1) \leq y_{1}(n) \exp \left[-r_{21}+a_{21}^{(1)}\left(A_{1}+\epsilon\right)-a_{22}^{(1)} y_{1}(n)\right], \\
n>N_{1} .
\end{array}
$$

Hence, using the maximum of $z(x)=x \exp (r-a x)$ as earlier, we obtain

$$
\limsup _{n \rightarrow+\infty} y_{1}(n) \leq \frac{1}{a_{22}^{(1)}} \exp \left[-r_{21}+a_{21}^{(1)}\left(A_{1}+\epsilon\right)-1\right] .
$$

Since $\epsilon$ is arbitrary, letting $\epsilon \rightarrow 0$ in (A.7) yields

$$
\limsup _{n \rightarrow+\infty} y_{1}(n) \leq \frac{1}{a_{22}^{(1)}} \exp \left[-r_{21}+a_{21}^{(1)} A_{1}-1\right] \triangleq M_{1} \text {. }
$$
that

Now, in view of inequality (9), we can choose $\epsilon_{1}>0$ such

$$
r_{11}-a_{12}^{(1)}\left(M_{1}+\epsilon_{1}\right)>0 .
$$

Further, noting (A.8) we have from the first equation of (7)

$$
x_{1}(n+1) \geq x_{1}(n) \exp \left[r_{11}-a_{11}^{(1)} x_{1}(n)-a_{12}^{(1)}\left(M_{1}+\epsilon_{1}\right)\right],
$$

for sufficiently large $n$. Using the inequality $\exp (x-1) \geq x$ which holds for $x>0$, together with the definition of $A_{1}$ (see (A.4)), we find

$$
a_{11}^{(1)} A_{1}=\exp \left(r_{11}-1\right) \geq r_{11}>r_{11}-a_{12}^{(1)}\left(M_{1}+\epsilon_{1}\right)>0,
$$

or

$$
\frac{a_{11}^{(1)} A_{1}}{r_{11}-a_{12}^{(1)}\left(M_{1}+\epsilon_{1}\right)}>1
$$

Inequalities (A.4) and (A.12) imply that Lemma 13 can be applied to (A.10). Thus, we have

$$
\begin{aligned}
& \liminf _{n \rightarrow+\infty} x_{1}(n) \\
& \geq \frac{r_{11}-a_{12}^{(1)}\left(M_{1}+\epsilon_{1}\right)}{a_{11}^{(1)}} \exp \left[r_{11}-a_{12}^{(1)}\left(M_{1}+\epsilon_{1}\right)-a_{11}^{(1)} A_{1}\right] .
\end{aligned}
$$

Again let $\epsilon_{1} \rightarrow 0$ in (A.13) and it follows that

$$
\begin{aligned}
& \liminf _{n \rightarrow+\infty} x_{1}(n) \\
& \geq \frac{r_{11}-a_{12}^{(1)} M_{1}}{a_{11}^{(1)}} \exp \left[r_{11}-a_{12}^{(1)} M_{1}-a_{11}^{(1)} A_{1}\right] \triangleq m_{1} .
\end{aligned}
$$

It is clear from (9) that $m_{1}>0$.

We will further show that $m_{1}<A_{1}$. From the derivation of (A.12), we have

$$
a_{11}^{(1)} A_{1}>r_{11}-a_{12}^{(1)} M_{1}
$$

that is, $r_{11}-a_{12}^{(1)} M_{1}-a_{11}^{(1)} A_{1}<0$, and so

$$
\exp \left[r_{11}-a_{12}^{(1)} M_{1}-a_{11}^{(1)} A_{1}\right]<1 .
$$

Therefore, from the definition of $m_{1}$ (see (A.14)) we find

$$
\begin{aligned}
m_{1} & =\frac{r_{11}-a_{12}^{(1)} M_{1}}{a_{11}^{(1)}} \exp \left[r_{11}-a_{12}^{(1)} M_{1}-a_{11}^{(1)} A_{1}\right] \\
& <\frac{r_{11}-a_{12}^{(1)} M_{1}}{a_{11}^{(1)}}<\frac{a_{11}^{(1)} A_{1}}{a_{11}^{(1)}}=A_{1} .
\end{aligned}
$$

In summary, combining (A.4), (A.14), and (A.17) we get

$$
0<m_{1} \leq \liminf _{n \rightarrow+\infty} x_{1}(n) \leq \limsup _{n \rightarrow+\infty} x_{1}(n) \leq A_{1} .
$$

In the following, we will establish the second inequality in Definition 1. In fact, we will prove that there exists $m_{3}>0$ such that

$$
\liminf _{n \rightarrow+\infty} y_{1}(n) \geq m_{3}
$$

Recalling that $m_{1}>0$ and noting inequality (10), we can choose $\epsilon_{2}>0$ sufficiently small such that $m_{1}-\epsilon_{2}>0$ and $-r_{21}+a_{21}^{(1)}\left(m_{1}-\epsilon_{2}\right)>0$. From the second equation of (7) and (A.14), we have

$$
y_{1}(n+1) \geq y_{1}(n) \exp \left[-r_{21}+a_{21}^{(1)}\left(m_{1}-\epsilon_{2}\right)-a_{22}^{(1)} y_{1}(n)\right],
$$

for sufficiently large $n$. Using the inequality $\exp (x-1) \geq x$ which holds, for $x>0$, and the fact that $m_{1}<A_{1}$, we find

$$
\begin{aligned}
a_{22}^{(1)} M_{1} & =\exp \left(-r_{21}+a_{21}^{(1)} A_{1}-1\right) \\
& \geq-r_{21}+a_{21}^{(1)} A_{1}>-r_{21}+a_{21}^{(1)} m_{1} \\
& >-r_{21}+a_{21}^{(1)}\left(m_{1}-\epsilon_{2}\right)>0 .
\end{aligned}
$$


Hence,

$$
\frac{a_{22}^{(1)} M_{1}}{-r_{21}+a_{21}^{(1)}\left(m_{1}-\epsilon_{2}\right)}>1
$$

which, together with (A.8), indicates that we can apply Lemma 13 to (A.20) to give

$$
\begin{aligned}
\liminf _{n \rightarrow+\infty} & y_{1}(n) \\
\geq & \frac{-r_{21}+a_{21}^{(1)}\left(m_{1}-\epsilon_{2}\right)}{a_{22}^{(1)}} \\
& \times \exp \left(-r_{21}+a_{21}^{(1)}\left(m_{1}-\epsilon_{2}\right)-a_{22}^{(1)} M_{1}\right) .
\end{aligned}
$$

Letting $\epsilon_{2} \rightarrow 0$, we get

$$
\begin{aligned}
& \liminf _{n \rightarrow+\infty} y_{1}(n) \\
& \geq \frac{-r_{21}+a_{21}^{(1)} m_{1}}{a_{22}^{(1)}} \exp \left(-r_{21}+a_{21}^{(1)} m_{1}-a_{22}^{(1)} M_{1}\right) \triangleq m_{3} .
\end{aligned}
$$

It is obvious from (10) that $m_{3}>0$.

We will further show that $m_{3}<M_{1}$. From (A.21), we have

$$
-r_{21}+a_{21}^{(1)} m_{1}-a_{22}^{(1)} M_{1}<0,
$$

and so

$$
\begin{aligned}
m_{3} & =\frac{-r_{21}+a_{21}^{(1)} m_{1}}{a_{22}^{(1)}} \exp \left(-r_{21}+a_{21}^{(1)} m_{1}-a_{22}^{(1)} M_{1}\right) \\
& <\frac{-r_{21}+a_{21}^{(1)} m_{1}}{a_{22}^{(1)}}<\frac{a_{22}^{(1)} M_{1}}{a_{22}^{(1)}}=M_{1} .
\end{aligned}
$$

Combining (A.8), (A.24), and (A.26), we get

$$
0<m_{3} \leq \liminf _{n \rightarrow+\infty} y_{1}(n) \leq \limsup _{n \rightarrow+\infty} y_{1}(n) \leq M_{1} .
$$

In view of (A.18) and (A.27), we have obtained both inequalities in Definition 1 and so the permanence of model (7) with initial values (8) is established. This completes the proof of Theorem 2 .

Proof of Theorem 4. Using a similar argument as in the proof of Theorem 2, we see that, under the assumptions (12) and (13), the following model

$$
\begin{aligned}
& x_{2}(n+1)=x_{2}(n) \exp \left[r_{12}-a_{11}^{(2)} x_{2}(n)-a_{12}^{(2)} y_{2}(n)\right] \\
& y_{2}(n+1)=y_{2}(n) \exp \left[-r_{22}+a_{21}^{(2)} x_{2}(n)-a_{22}^{(2)} y_{2}(n)\right]
\end{aligned}
$$

with initial values $x_{2}(0)=x_{2}>0, y_{2}(0)=y_{2}>0$ is permanent. In fact, analogous to (A.18) and (A.27), we have

$$
\begin{aligned}
& 0<m_{2} \leq \liminf _{n \rightarrow+\infty} x_{2}(n) \leq \limsup _{n \rightarrow+\infty} x_{2}(n) \leq A_{2}, \\
& 0<m_{4} \leq \liminf _{n \rightarrow+\infty} y_{2}(n) \leq \limsup _{n \rightarrow+\infty} y_{2}(n) \leq M_{2},
\end{aligned}
$$

where $m_{2}, A_{2}$, and $M_{2}$ are given in Theorem 4 and

$$
m_{4}=\frac{-r_{22}+a_{21}^{(2)} m_{2}}{a_{22}^{(2)}} \exp \left(-r_{22}+a_{21}^{(2)} m_{2}-a_{22}^{(2)} M_{2}\right) \text {. }
$$

Coupling with Theorem 2, the permanence of model (3) with initial values (5) follows immediately. This completes the proof of Theorem 4 .

Proof of Theorem 5. Consider the following auxiliary system:

$$
\begin{aligned}
& u(n+1)=u(n) \exp \left[r_{11}-a_{11}^{(1)} u(n)-a_{12}^{(1)} v(n)-d_{12} u(n)\right], \\
& v(n+1)=v(n) \exp \left[-r_{21}+a_{21}^{(1)} u(n)-a_{22}^{(1)} v(n)\right]
\end{aligned}
$$

with initial values $u(0)=x_{1}>0, v(0)=y_{1}>0$.

Comparing (7) and (A.31), it follows from Theorem 2 that model (A.31) is permanent under conditions (15) and (16). Therefore, there exist positive constants $m_{1}^{(1)}$ and $m_{1}^{(2)}$ such that

$$
\liminf _{n \rightarrow+\infty} u(n) \geq m_{1}^{(1)}, \quad \liminf _{n \rightarrow+\infty} v(n) \geq m_{1}^{(2)} .
$$

From the first two equations of (4), it is clear that, for any $n \in \mathcal{N}$, where $\mathcal{N}$ represents the set of all positive integers, we have $x_{1}(n) \geq u(n)$ and $y_{1}(n) \geq v(n)$. Hence,

$$
\begin{aligned}
& \liminf _{n \rightarrow+\infty} x_{1}(n) \geq \liminf _{n \rightarrow+\infty} u(n) \geq m_{1}^{(1)}, \\
& \liminf _{n \rightarrow+\infty} y_{1}(n) \geq \liminf _{n \rightarrow+\infty} v(n) \geq m_{1}^{(2)} .
\end{aligned}
$$

Next, we consider the following auxiliary system:

$$
\begin{aligned}
& p(n+1)=p(n) \exp \left[r_{12}-a_{11}^{(2)} p(n)-a_{12}^{(2)} q(n)-d_{21} p(n)\right] \\
& q(n+1)=q(n) \exp \left[-r_{22}+a_{21}^{(2)} p(n)-a_{22}^{(2)} q(n)\right]
\end{aligned}
$$

with initial values $p(0)=x_{2}>0, q(0)=y_{2}>0$. By a similar argument as above, we see that (A.34) is permanent under conditions (17) and (18). Thus, there exist positive constants $m_{2}^{(1)}$ and $m_{2}^{(2)}$ such that

$$
\liminf _{n \rightarrow+\infty} p(n) \geq m_{2}^{(1)}, \quad \liminf _{n \rightarrow+\infty} q(n) \geq m_{2}^{(2)} .
$$

In view of the last two equations of (4), we have $x_{2}(n) \geq p(n)$ and $y_{2}(n) \geq q(n)$, for any $n \in \mathcal{N}$. It follows that

$$
\begin{aligned}
& \liminf _{n \rightarrow+\infty} x_{2}(n) \geq \liminf _{n \rightarrow+\infty} p(n) \geq m_{2}^{(1)}, \\
& \liminf _{n \rightarrow+\infty} y_{2}(n) \geq \liminf _{n \rightarrow+\infty} q(n) \geq m_{2}^{(2)} .
\end{aligned}
$$

To complete the proof, in the following we will prove that there exist positive constants $M_{i}^{(j)}, i, j \in\{1,2\}$, such that

$$
\begin{array}{ll}
\limsup _{n \rightarrow+\infty} x_{1}(n) \leq M_{1}^{(1)}, & \limsup _{n \rightarrow+\infty} y_{1}(n) \leq M_{1}^{(2)}, \\
\limsup _{n \rightarrow+\infty} x_{2}(n) \leq M_{2}^{(1)}, & \limsup _{n \rightarrow+\infty} y_{2}(n) \leq M_{2}^{(2)} .
\end{array}
$$


Suppose the contrary; there exists no such $M_{1}^{(1)}>0$ to satisfy $\lim \sup _{n \rightarrow+\infty} x_{1}(n) \leq M_{1}^{(1)}$, and then we can select a subsequence $\left\{x_{1}\left(n_{k}\right)\right\}_{k=1}^{\infty}$ such that

$$
\lim _{k \rightarrow+\infty} x_{1}\left(n_{k}\right)=+\infty
$$

Multiplying the first equation with the third equation of (4), we get

$$
\begin{aligned}
& x_{1}(n+1) x_{2}(n+1) \\
& =x_{1}(n) x_{2}(n) \exp \left[r_{11}+r_{12}-a_{11}^{(1)} x_{1}(n)-a_{12}^{(1)} y_{1}(n)\right. \\
& \left.\quad-a_{11}^{(2)} x_{2}(n)-a_{12}^{(2)} y_{2}(n)\right] \\
& \leq x_{1}(n) x_{2}(n) \exp \left[r_{11}+r_{12}-a_{11}^{(1)} x_{1}(n)-a_{11}^{(2)} x_{2}(n)\right] .
\end{aligned}
$$

Note that the two-variable function $f(x, y)=x y \exp (a-b x-$ $c y)$ is a bounded function in the first quadrant $\{(x, y) \mid x>$ $0, y>0\}$ provided that $a, b, c>0$. Hence, it follows from (A.39) that $\limsup _{n \rightarrow+\infty} x_{1}(n) x_{2}(n)$ is finite. Noting (A.38), this implies $\lim _{k \rightarrow+\infty} x_{2}\left(n_{k}\right)=0$, which is a contradiction to (A.36). This shows that $\lim \sup _{n \rightarrow+\infty} x_{1}(n) \leq M_{1}^{(1)}$. Similarly, we can prove that $\lim _{\sup _{n \rightarrow+\infty}} x_{2}(n) \leq M_{2}^{(1)}$. Summarizing, we have established the following inequalities:

$$
\limsup _{n \rightarrow+\infty} x_{1}(n) \leq M_{1}^{(1)}, \quad \limsup _{n \rightarrow+\infty} x_{2}(n) \leq M_{2}^{(1)} .
$$

Now, from the second equation of (4) and (A.40), we have, for sufficiently small $\epsilon_{3}>0$,

$$
\begin{aligned}
& y_{1}(n+1) \\
& \leq y_{1}(n) \exp \left[-r_{21}+a_{21}^{(1)}\left(M_{1}^{(1)}+\epsilon_{3}\right)-a_{22}^{(1)} y_{1}(n)\right. \\
& \left.+\alpha d_{21}\left(M_{2}^{(1)}+\epsilon_{3}\right)\right],
\end{aligned}
$$

for sufficiently large $n$. Noting that the maximum of the function $z(x)=x \exp (r-a x)$ where $a>0$ in the interval $[0,+\infty)$ is $z_{\max }=(1 / a) \exp (r-1)$, it follows from (A.41) that

$$
\begin{aligned}
& \limsup _{n \rightarrow+\infty} y_{1}(n) \\
& \leq \frac{1}{a_{22}^{(1)}} \exp \left[-r_{21}+a_{21}^{(1)}\left(M_{1}^{(1)}+\epsilon_{3}\right)+\alpha d_{21}\left(M_{2}^{(1)}+\epsilon_{3}\right)-1\right] .
\end{aligned}
$$

Letting $\epsilon_{3} \rightarrow 0$ in the above inequality, we obtain

$$
\begin{aligned}
& \limsup _{n \rightarrow+\infty} y_{1}(n) \\
& \leq \frac{1}{a_{22}^{(1)}} \exp \left[-r_{21}+a_{21}^{(1)} M_{1}^{(1)}+\alpha d_{21} M_{2}^{(1)}-1\right] \triangleq M_{1}^{(2)}
\end{aligned}
$$

Likewise, a similar deduction as above gives

$$
\begin{aligned}
& \limsup _{n \rightarrow+\infty} y_{2}(n) \\
& \leq \frac{1}{a_{22}^{(2)}} \exp \left[-r_{22}+a_{21}^{(2)} M_{2}^{(1)}+\beta d_{12} M_{1}^{(1)}-1\right] \triangleq M_{2}^{(2)} .
\end{aligned}
$$

Combining (A.40), (A.43), and (A.44), we have shown that (A.37) holds. The permanence of model (4) with initial values (5) now follows from (A.33), (A.36), and (A.37). This completes the proof of Theorem 5 .

\section{Conflict of Interests}

The authors declare that there is no conflict of interests regarding the publication of this paper.

\section{Acknowledgments}

The authors would like to thank the reviewers for their comments which help to improve the paper. The research is sponsored by Jiangsu Overseas Research \& Training Program.

\section{References}

[1] P. Schuster, K. Sigmund, and R. Wolff, "Dynamical systems under constant organization. III. Cooperative and competitive behavior of hypercycles," Journal of Differential Equations, vol. 32, no. 3, pp. 357-368, 1979.

[2] C. Wu and J. Cui, "Global dynamics of discrete competitive models with large intrinsic growth rates," Discrete Dynamics in Nature and Society, vol. 2009, Article ID 710353, 15 pages, 2009.

[3] C. Wu, "Permanence and stable periodic solution for a discrete competitive system with multidelays," Advances in Difference Equations, vol. 2009, Article ID 375486, 12 pages, 2009.

[4] Z. Zhou and X. Zou, "Stable periodic solutions in a discrete periodic logistic equation," Applied Mathematics Letters, vol. 16, no. 2, pp. 165-171, 2003.

[5] C. Çelik and O. Duman, "Allee effect in a discrete-time predatorprey system," Chaos, Solitons and Fractals, vol. 40, no. 4, pp. 1956-1962, 2009.

[6] J. Baštinec, L. Berezansky, J. Diblík, and Z. Šmarda, "On a delay population model with a quadratic nonlinearity without positive steady state," Applied Mathematics and Computation, vol. 227, pp. 622-629, 2014.

[7] L. Berezansky, J. Baštinec, J. Diblík, and Z. Šmarda, "On a delay population model with quadratic nonlinearity," Advances in Difference Equations, vol. 2012, article 230, 2012.

[8] V. A. A. Jansen and K. Sigmund, "Shaken not stirred: on permanence in ecological communities," Theoretical Population Biology, vol. 54, no. 3, pp. 195-201, 1998.

[9] A. Hastings, "Complex interactions between dispersal and dynamics: lessons from coupled logistic equations," Ecology, vol. 74, no. 5, pp. 1362-1372, 1993.

[10] M. D. Holland and A. Hastings, "Strong effect of dispersal network structure on ecological dynamics," Nature, vol. 456, no. 7223, pp. 792-795, 2008. 
[11] Y. Kim, O. Kwon, and F. Li, "Evolution of dispersal toward fitness," Bulletin of Mathematical Biology, vol. 75, no. 12, pp. 2474-2498, 2013.

[12] S. A. Levin, "Dispersal and population interactions," American Naturalist, vol. 108, pp. 207-228, 1974.

[13] Y. Lou and F. Lutscher, "Evolution of dispersal in open advective environments," Journal of Mathematical Biology, 2013.

[14] N. Moquet, M. F. Hoopes, and P. Amarasekare, "The world is patchy and heterogeneous! Trade-off and source-sink dynamics in competitive metacommunities," in Metacommunities: Spatial Dynamics and Ecological Communities, chapter 10, pp. 237-262, University of Chicago Press, Chicago, Ill, USA, 2005.

[15] S. J. Schreiber and T. P. Killingback, "Spatial heterogeneity promotes coexistence of rock-paper-scissors metacommunities," Theoretical Population Biology, vol. 86, pp. 1-11, 2013.

[16] X. Zhang and W. D. Wang, "Importance of dispersal adaptations of two competitive populations between patches," Ecological Modelling, vol. 222, no. 1, pp. 11-20, 2011.

[17] http://www.china.com.cn/city/txt/2007-07/02/content8466486 .htm.

[18] F. Kong, Studies on the adaptability and behavior time assignments of Tibtan antelope using wild life passageways of QinghaiTibet railway, [Master dissertation], Northwest University, Shaanxi, China, 2009, (Chinese).

[19] J. Cui and L. Chen, "Permanence and extinction in logistic and Lotka-Volterra systems with diffusion," Journal of Mathematical Analysis and Applications, vol. 258, no. 2, pp. 512-535, 2001.

[20] J. Cui, Y. Takeuchi, and Z. Lin, "Permanence and extinction for dispersal population systems," Journal of Mathematical Analysis and Applications, vol. 298, no. 1, pp. 73-93, 2004.

[21] Z. Lu and Y. Zhou, Advances in Mathematical Biology, Science Press, Beijing, China, 2006, (Chinese).

[22] C. Wu and J. Cui, "Permanence for a delayed discrete predatorprey model with prey dispersal," International Journal of Biomathematics, vol. 2, no. 3, pp. 311-320, 2009.

[23] L. Zhang and Z. Teng, "Permanence for a delayed periodic predator-prey model with prey dispersal in multi-patches and predator density-independent," Journal of Mathematical Analysis and Applications, vol. 338, no. 1, pp. 175-193, 2008.

[24] E. Braverman and D. Kinzebulatov, "On linear perturbations of the Ricker model," Mathematical Biosciences, vol. 202, no. 2, pp. 323-339, 2006.

[25] Y. Kang, D. Armbruster, and Y. Kuang, "Dynamics of a plantherbivore model," Journal of Biological Dynamics, vol. 2, no. 2 , pp. 89-101, 2008.

[26] G. Sun, G. Zhang, and Z. Jin, "Dynamic behavior of a discrete modified Ricker and Beverton-Holt model," Computers and Mathematics with Applications, vol. 57, no. 8, pp. 1400-1412, 2009.

[27] Y. Chow and S. R. Jang, "Coexistence in a discrete competition model with dispersal," Journal of Difference Equations and Applications, vol. 19, no. 4, pp. 615-632, 2013.

[28] J. A. L. Silva and F. T. Giordani, "Density-dependent migration and synchronism in metapopulations," Bulletin of Mathematical Biology, vol. 68, no. 2, pp. 451-465, 2006.

[29] J. A. L. Silva and F. T. Giordani, "Density-dependent dispersal in multiple species metapopulations," Mathematical Biosciences and Engineering, vol. 5, no. 4, pp. 843-857, 2008.

[30] A. Yakubu, "Asynchronous and synchronous dispersals in spatially discrete population models," SIAM Journal on Applied Dynamical Systems, vol. 7, no. 2, pp. 284-310, 2008.
[31] R. M. May, "Nonlinear problems in ecology," in Chaotic Behavior of Deterministic Systems, G. Iooss, R. Helleman, and R. Stora, Eds., North-Holland, Amsterdam, The Netherlands, 1983.

[32] E. W. Ricker, "Stock and recruitment," Journal of the Fisheries Research Board of Canada, vol. 11, no. 5, pp. 559-623, 1954.

[33] A. Hastings, Population Biology: Concepts and Models, Springer, New York, NY, USA, 1996.

[34] H. L. Smith, Monotone Dynamical Systems: An Introduction to the Theory of Competitive and Cooperatiive Systems, vol. 41 of Mathematical Surveys and Monographs, American Mathematical Society, Providence, RI, USA, 1995.

[35] Z. Lu and W. Wang, "Permanence and global attractivity for Lotka-Volterra difference systems," Journal of Mathematical Bio$\log y$, vol. 39, no. 3, pp. 269-282, 1999.

[36] W. Wang and Z. Lu, "Global stability of discrete models of Lotka-Voltera type," Nonlinear Analysis: Theory, Methods \& Applications, vol. 35, no. 8, pp. 1019-1030, 1999.

[37] M. Y. Li and Z. Shuai, "Global-stability problem for coupled systems of differential equations on networks," Journal of Differential Equations, vol. 248, no. 1, pp. 1-20, 2010.

[38] L. Zu, D. Q. Jiang, and F. Q. Jiang, "Existence, stationary distribution, and extinction of predator-prey system of prey dispersal with stochastic perturbation," Abstract and Applied Analysis, vol. 2012, Article ID 547152, 24 pages, 2012.

[39] F. S. Berezovskaya, B. J. Song, and C. Castillo-Chavez, "Role of prey dispersal and refuges on predator-prey dynamics," SIAM Journal on Applied Mathematics, vol. 70, no. 6, pp. 1821-1839, 2010.

[40] G. E. Forrester, "Influences of predatory fish on the drift dispersal and local density of stream insects," Ecology, vol. 75, no. 5, pp. 1208-1218, 1994.

[41] L. J. S. Allen, "Persistence and extinction in single-species reaction-diffusion models," Bulletin of Mathematical Biology, vol. 45, no. 2, pp. 209-227, 1983.

[42] C. Wu, "On the global asymptotic behavior of a discrete predator-prey model," Advances and Applications in Mathematical Sciences, vol. 10, no. 2, pp. 143-157, 2011.

[43] X. Yang, "Uniform persistence and periodic solutions for a discrete predator-prey system with delays," Journal of Mathematical Analysis and Applications, vol. 316, no. 1, pp. 161-177, 2006. 


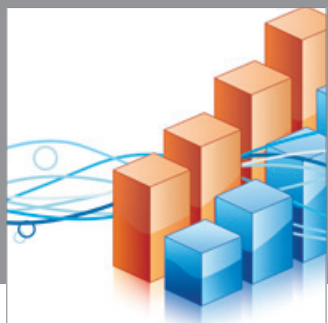

Advances in

Operations Research

mansans

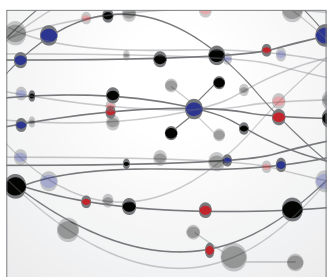

The Scientific World Journal
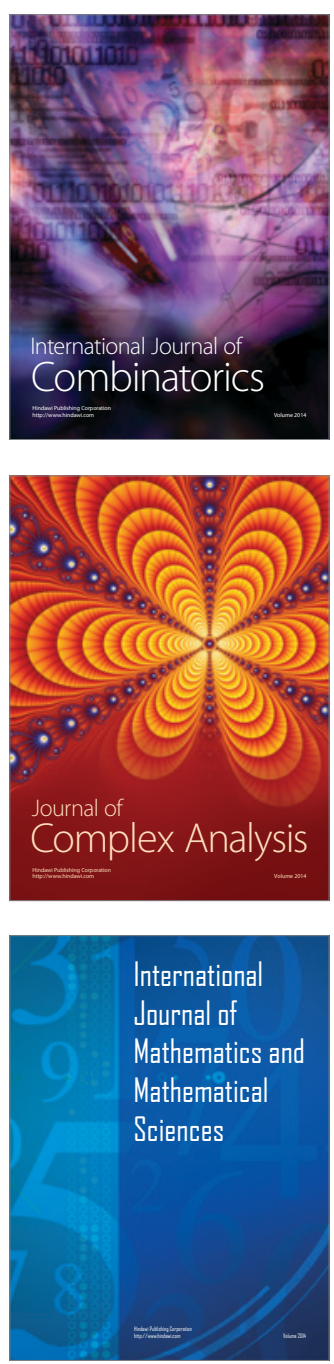
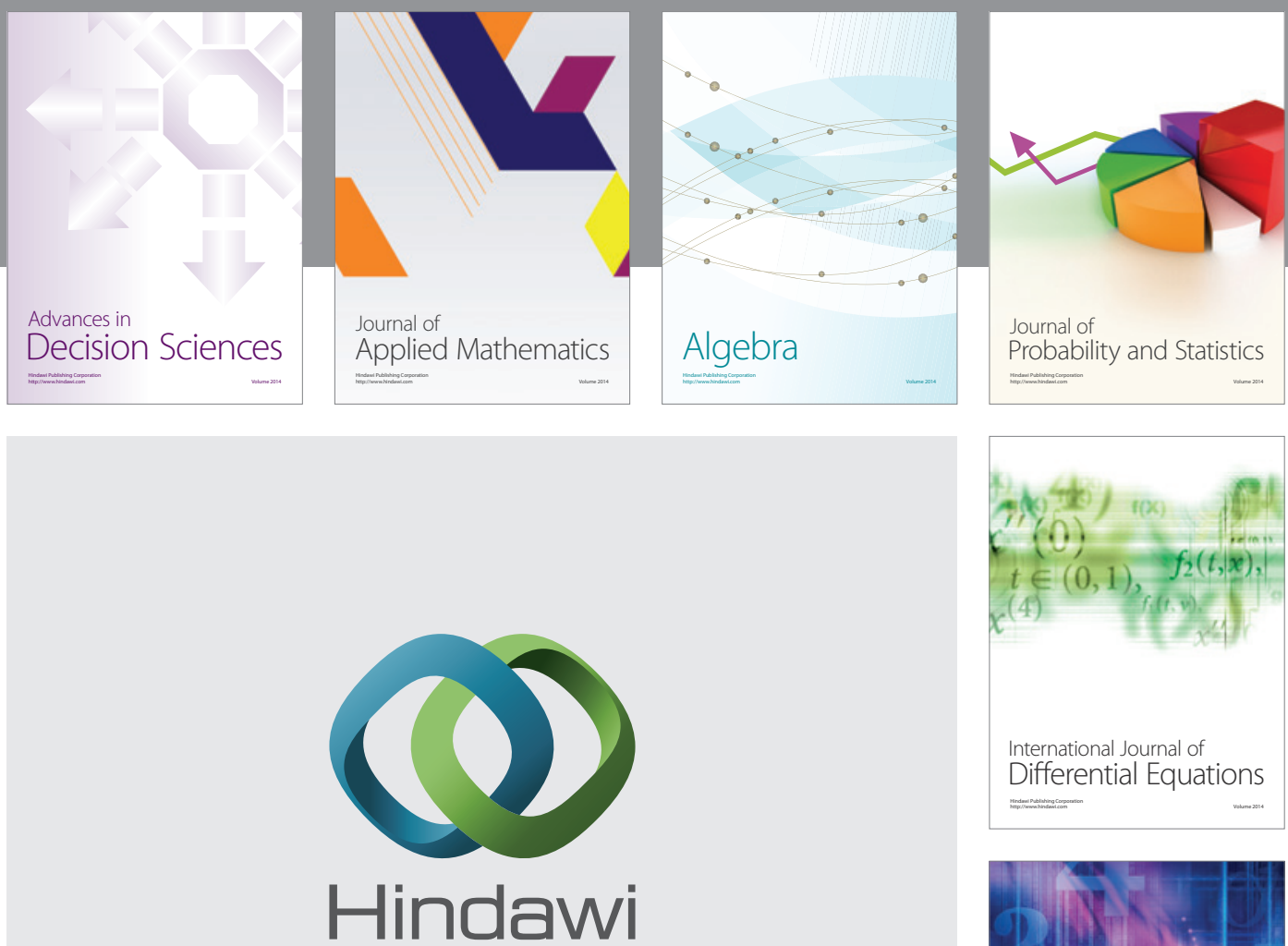

Submit your manuscripts at http://www.hindawi.com
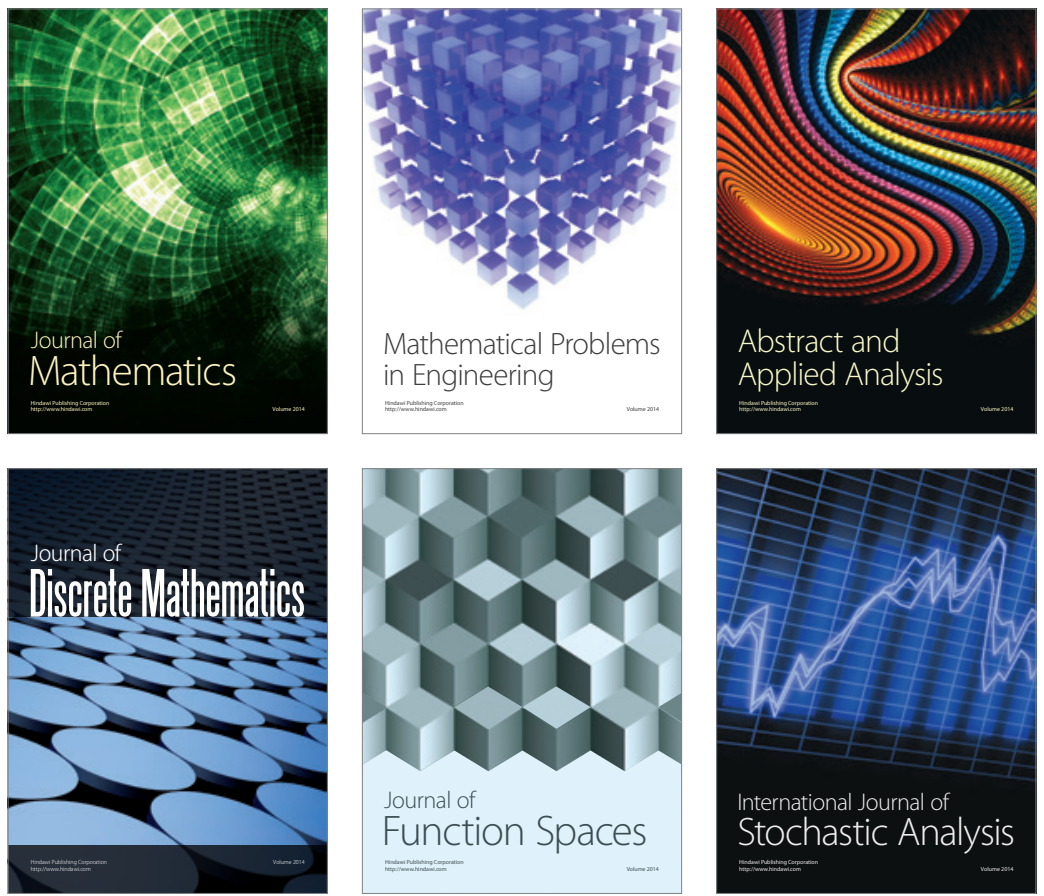

Journal of

Function Spaces

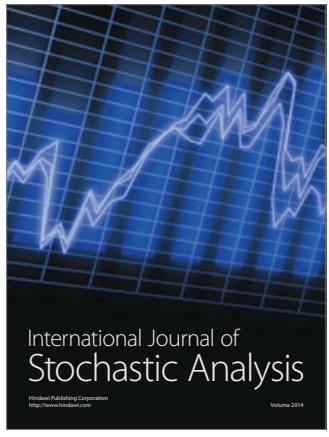

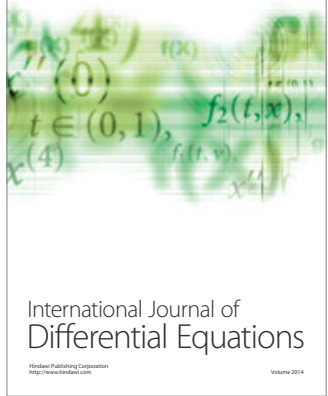
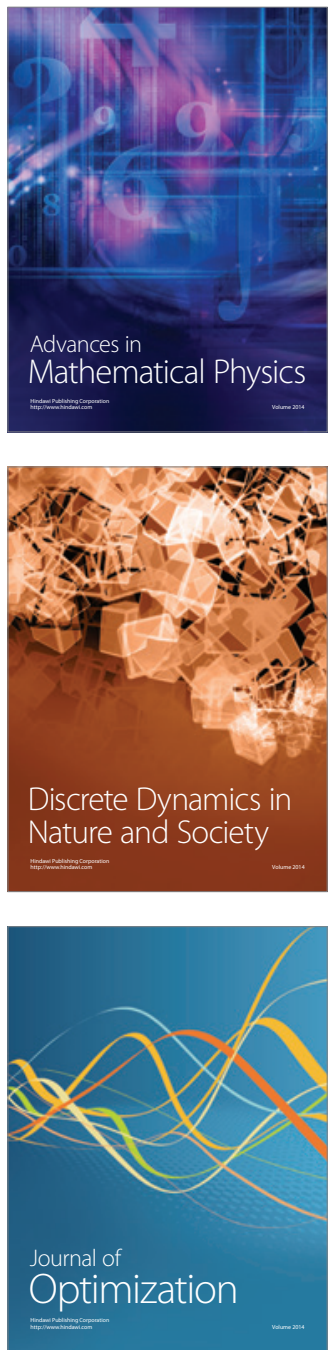